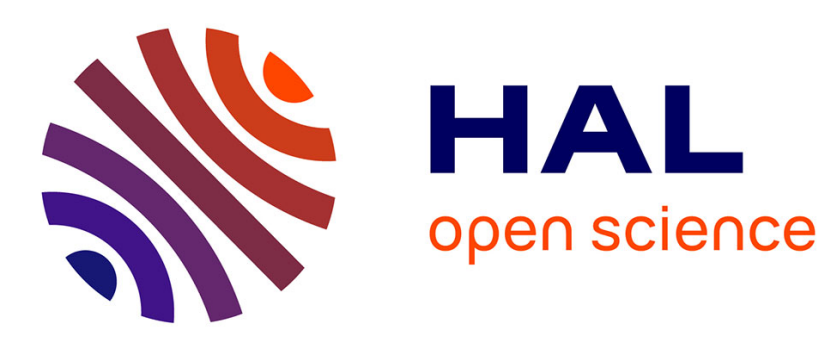

\title{
Phase-field modeling of a fluid-driven fracture in a poroelastic medium
}

\author{
Andro Mikelic, M.F. Wheeler, Thomas Wick
}

\section{To cite this version:}

Andro Mikelic, M.F. Wheeler, Thomas Wick. Phase-field modeling of a fluid-driven fracture in a poroelastic medium. Computational Geosciences, 2015, 19 (6), pp.1171-1195. 10.1007/s10596-0159532-5 . hal-01202370

\section{HAL Id: hal-01202370 \\ https://hal.science/hal-01202370}

Submitted on 20 Sep 2015

HAL is a multi-disciplinary open access archive for the deposit and dissemination of scientific research documents, whether they are published or not. The documents may come from teaching and research institutions in France or abroad, or from public or private research centers.
L'archive ouverte pluridisciplinaire HAL, est destinée au dépôt et à la diffusion de documents scientifiques de niveau recherche, publiés ou non, émanant des établissements d'enseignement et de recherche français ou étrangers, des laboratoires publics ou privés. 


\title{
Phase-field modeling of a fluid-driven fracture in a poroelastic medium
}

\author{
Andro Mikelić ${ }^{\dagger}$ \\ Mary F. Wheeler $\ddagger$ \\ Thomas Wick $\S$
}

In this paper we present a phase field model for a fluid-driven fracture in a poroelastic medium. In our previous work, the pressure was assumed given. Here we consider a fully coupled system where the pressure field is determined simultaneously with the displacement and the phase field. To the best of our knowledge, such a model is new in the literature. The mathematical model consists of a linear elasticity system with fading elastic moduli as the crack grows, which is coupled with an elliptic variational inequality for the phase field variable and with the pressure equation containing the phase field variable in its coefficients. The convex constraint of the variational inequality assures the irreversibility and entropy compatibility of the crack formation. The phase field variational inequality contains quadratic pressure and strain terms, with coefficients depending on the phase field unknown. We establish existence of a solution to the incremental problem through convergence of a finite dimensional approximation. Furthermore, we construct the corresponding Lyapunov functional that is linked to the free energy. Computational results are provided that demonstrate the effectiveness of this approach in treating fluid-driven fracture propagation.

Keywords: Hydraulic fracturing, Phase field formulation, Nonlinear elliptic-parabolic system, Computer simulations, Poroelasticity

\footnotetext{
${ }^{*}$ The research by A. Mikelic, M. F. Wheeler and T. Wick was partially supported by ConocoPhillips grant UTA13-001170 AMD 1 and Statoil grant UTA13-000884: WR DTD 2.13.14. A. Mikelic and T. Wick would like to thank Institute for Computational Engineering and Science (ICES), UT Austin for hospitality during their visits in February, June and August 2015 and support through a JT Oden fellowship. M. F. Wheeler was also supported by Aramco grant UTA11-000320; 1ST and T. Wick by the Austrian Academy of Sciences.

†Université de Lyon, CNRS UMR 5208, Université Lyon 1, Institut Camille Jordan, 43, blvd. du 11 novembre 1918, 69622 Villeurbanne Cedex, France

${ }^{\ddagger}$ Center for Subsurface Modeling, The Institute for Computational Engineering and Sciences, The University of Texas at Austin, Austin, Texas 78712, USA

${ }^{\S}$ RICAM, Austrian Academy of Sciences Altenberger Str. 694040 Linz, Austria and Fakultät für Mathematik, Lehrstuhl M17, Technische Universität München, 85748 Garching bei München, Germany
} 


\section{Introduction}

The coupling of flow and geomechanics in porous media is a major research topic in energy and environmental modeling. Of specific interest is induced hydraulic fracturing or hydrofracturing commonly known as fracking. This technique is used to release petroleum and natural gas that includes shale gas, tight gas, and coal seam gas for extraction. Here fracking creates fractures from a wellbore drilled into reservoir rock formations. According to the US National Petroleum Council $95 \%$ of oil and gas wells drilled today are hydraulically fractured. Clearly there are economic benefits of extracting vast amounts of formerly inaccessible hydrocarbons. In addition, there are environmental benefits of producing natural gas, much of which is produced in the United States from fracking. Opponents to fracking point to environmental impacts such as contamination of ground water, risks to air quality, migration of fracturing chemical and surface contamination from spills to name a few. For this reason, hydraulic fracturing is being heavily scrutinized resulting in the need for accurate and robust mathematical and computational models for treating fluid field fractures surrounded by a poroelastic medium.

Even in the most basic formulation, hydraulic fracturing is complicated to model since it involves the coupling of (i) mechanical deformation; (ii) the flow of fluids within the fracture and in the reservoir; (iii) fracture propagation. Generally, rock deformation is modeled using the theory of linear elasticity which can be represented by an integral equation that determines a relationship between fracture width and the fluid pressure. Fluid flow in the fracture is modeled using lubrication theory that relates fluid flow velocity, fracture width and the gradient of pressure. Fluid flow in the reservoir is modeled as a Darcy flow and the respective fluids are coupled through a leakage term. The criterion for fracture propagation is usually given by the conventional energy-release rate approach of linear elastic fracture mechanics (LEFM) theory; that is the fracture propagates if the stress intensity factor at the tip matches the rock toughness. Detailed discussion of the development of hydraulic fracturing models for use in petroleum engineering can be found in [1] and in mechanical engineering and hydrology in [18, [5, [11] and in references therein.

The goal of this paper is to develop a complete and robust phase field model for a fluid-driven fracture propagation in a poroelastic medium with the solid structure displacement, pressure and the phase-field variable as unknowns. We remark that in our preceding papers [15], 16] and [17, either pressure or displacements were assumed to be given.

The novelties in this coupled approach are:

- Fracture propagation in a poroelastic medium rather than in an elastic medium;

- Solving a fluid equation inside the fracture interacting with the fluid in the bulk poroelastic medium;

- Constructing the appropriate Lyapunov functional for the coupled three-field (displacementphase field-pressure) problem, which is linked to the phase-field modified free energy.

- Establishing mathematical stability of the three-field (displacement-phase field-pressure) equation, through constructing the corresponding Lyapunov functional and demonstrating effectiveness by providing several representative numerical tests.

The outline of our paper is as follows: In Section 2 we recall the basic equations of the sharp interface mesoscopic model and make some comments on the known phase-field approaches in the fracture mechanics. In Section 3 , we present a detailed phase-field modeling of the quasistatic fracture propagation in a poroelastic medium. In Section 4 we introduce an incremental formulation of a phase-field model for a pressurized crack from which we derive a Lyapunov functional. In Section 5 , 
we present a mathematical analysis of the incremental problem. In Section 6, a numerical formulation is briefly described. Finally in Section 7 we provide several numerical experiments demonstrating the capabilities of our proposed model.

\section{Fundamental Equations}

In order to fix ideas we address the simplest model of real applied importance, namely, the quasi-static single phase Biot system. We start by introducing the geometry.

\subsection{Geometry}

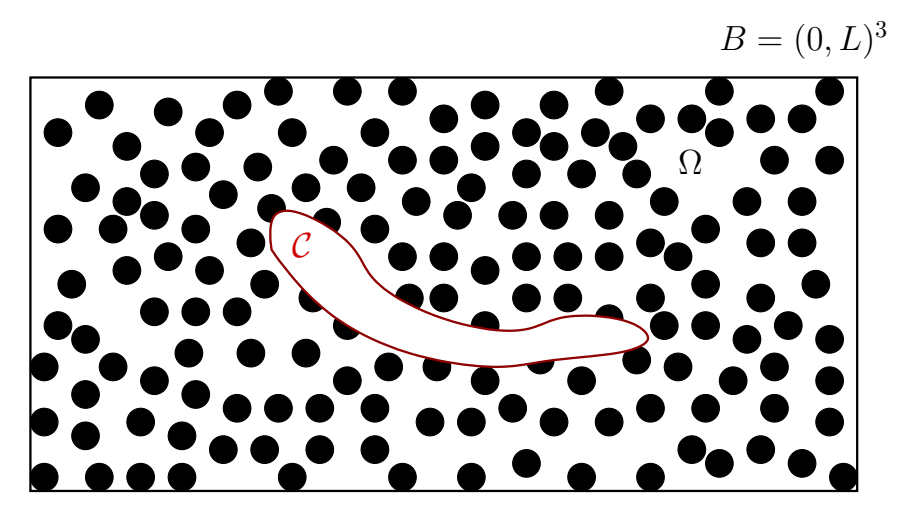

Figure 1: A crack $\mathcal{C}$ embedded in a porous medium. Here, the dimensions of the crack are assumed to be much larger than the pore scale size (black dots) of the porous medium.

Let $\mathcal{C}$ denote any open set homeomorphic to an ellipsoid in $\mathbb{R}^{3}$ (a crack set). Its boundary is a closed surface $\partial \mathcal{C}$. In most applications $\mathcal{C}$ is a curved $3 \mathrm{D}$ domain, with one dimension significantly smaller than the dominant two. An example is a penny shape crack.

The crack set $\mathcal{C}$ is surrounded by the poroelastic domain $\Omega=B \backslash \overline{\mathcal{C}}$, where $B=(0, L)^{3} \supset \supset \mathcal{C}$.

In many fracture propagation references, the $\operatorname{crack} \mathcal{C}$ is considered to be a lower dimensional manifold and the lubrication theory is applied to describe the fluid flow (see e.g. [1] and [10]). We remark that $3 \mathrm{D}$ flow in $\mathcal{C}$ can be reconstructed from a lower dimensional lubrication approximation as described in [17. Nevertheless, we consider $\mathcal{C}$ as a 3D domain and use its particular geometry only when discussing the stress interface conditions.

The boundary of $B=(0, L)^{3}$ is denoted by $\partial B$, which is divided into open $2 \mathrm{D}$ surfaces $\partial_{D} B$ and $\partial_{N} B$, with smooth boundaries, in the way that $\partial B=\overline{\partial_{D} B} \cup \overline{\partial_{N} B}$. On $\partial_{D} B$ Dirichlet boundary conditions will be imposed and on $\partial_{N} B$ Neumann conditions, respectively. We assume that meas $\left(\partial_{D} B\right)>0$. Boundary conditions on $\partial_{D} B$ for the Biot system involve displacements and the normal pressure fluxes and on $\partial_{N} \Omega$ traction as well as pressure.

\subsection{Biot equations}

The quasi-static Biot equations (see e.g. [21]) are an elliptic-parabolic system of PDEs, valid in the poroelastic domain $\Omega$, where for every $t \in(0, T)$ we have

$$
\begin{gathered}
\sigma^{p o r}-\sigma_{0}=\mathcal{G} e(\mathbf{u})-\alpha p_{R} I ; \quad-\operatorname{div}\left(\sigma^{p o r}\right)=\rho_{b} \mathbf{g} \\
\partial_{t}\left(\frac{1}{M} p_{R}+\operatorname{div}(\alpha \mathbf{u})\right)+\operatorname{div}\left(\frac{\mathcal{K}_{R}}{\eta_{R}}\left(\rho_{R} \mathbf{g}-\nabla p_{R}\right)\right)=q,
\end{gathered}
$$


where $\sigma_{0}$ is the reference state total stress and $q$ represents source terms. $\mathcal{G}$ is a constant symmetric positive definite rank-4 tensor. In our examples $(7.1)$ and $(7.2)$ we model the stress $\mathcal{G} e(u)$ using a linear stress-strain relationship:

$$
\mathcal{G} e(\mathbf{u})=2 \mu e(\mathbf{u})+\lambda \operatorname{tr}(e(\mathbf{u})) I,
$$

where $I$ is the identity matrix, $\operatorname{tr}(\cdot)$ the trace operator, and $\mu$ and $\lambda$ are Lamé's parameters, which are linked to Young's modulus $E$ and Poisson's ratio $\nu_{s}$ by $\mu=E /\left(2\left(1+\nu_{s}\right)\right)$ and $\lambda=E \nu_{s} /\left(\left(1+\nu_{s}\right)(1+\right.$ $\left.\left.2 \nu_{s}\right)\right)$.

\begin{tabular}{|l|l|l|}
\hline \hline$S Y M B O L$ & QUANTITY & UNITY \\
\hline $\mathbf{u}$ & displacement & $\mathrm{m}$ \\
\hline$p$ & fluid pressure & $\mathrm{Pa}$ \\
\hline$\sigma^{\text {por }}$ & total poroelasticity tensor & $\mathrm{Pa}$ \\
\hline$e(\mathbf{u})=\left(\nabla \mathbf{u}+\nabla^{\tau} \mathbf{u}\right) / 2$ & linearized strain tensor & dimensionless \\
\hline $\mathcal{K}_{R}$ & permeability of the poroelastic domain & Darcy \\
\hline $\mathcal{K}_{F}$ & fracture permeability & Darcy \\
\hline$\alpha$ & Biot's coefficient & dimensionless \\
\hline$\rho_{b}$ & bulk density & $\mathrm{kg} / \mathrm{m}^{3}$ \\
\hline$\eta_{F}$ & fracture fluid viscosity & $\mathrm{kg} / \mathrm{m} \mathrm{sec}$ \\
\hline$\eta_{R}$ & pore fluid viscosity & $\mathrm{kg} / \mathrm{m} \mathrm{sec}$ \\
\hline$M$ & Biot's modulus & $\mathrm{Pa}$ \\
\hline$c_{F}$ & fracture fluid compressibility & $1 / \mathrm{Pa}$ \\
\hline $\mathcal{G}$ & Gassman rank-4 tensor & $\mathrm{Pa}$ \\
\hline$\rho_{F}$ & reference state fracture fluid density & $\mathrm{kg} / \mathrm{m}^{3}$ \\
\hline$\rho_{R}$ & reference state pore fluid density & $\mathrm{kg} / \mathrm{m}^{3}$ \\
\hline $\mathbf{g}$ & gravity & $\mathrm{m} / \mathrm{sec}^{2}$ \\
\hline
\end{tabular}

Table 1: Unknowns and effective coefficients

The important parameters and unknowns are listed in the Table 1 .

Due to the fracture size, the relative velocity satisfies Darcy's law

$$
\mathbf{v}_{F}=\frac{\mathcal{K}_{F}}{\eta_{F}}\left(\mathbf{G}-\nabla p_{F}\right) .
$$

Note that for a penny shaped $\mathcal{C}$ the dominant velocity part is two-dimensional. An explicit expression for the permeability $\mathcal{K}_{F}$ as a function of the fracture shape is derived in [17]. The term $\mathbf{G}$ is a linear function of the gravity and leakage (see [17, Sec 2.3 for details). In the fracture $\mathcal{C}$, the pressure satisfies the mass conservation equation

$$
c_{F} \partial_{t} p+\operatorname{div}\left(\frac{\mathcal{K}_{F}}{\eta_{F}}(\mathbf{G}-\nabla p)\right)=q .
$$

Modeling the moving interface $\partial \mathcal{C}$ is delicate. We impose the following interface conditions

$$
\begin{gathered}
p_{R}=p_{F} \quad \text { and } \quad \sigma^{p o r} \mathbf{n}=-p_{F} \mathbf{n} \quad \text { on the moving interface } \partial \mathcal{C} \\
\mathbf{v}_{F} \cdot \mathbf{n}=\mathbf{v}_{R} \cdot \mathbf{n}=\frac{\mathcal{K}_{R}}{\eta_{R}}\left(\rho_{R} \mathbf{g}-\nabla p_{R}\right) \cdot \mathbf{n} \quad \text { on the moving interface } \partial \mathcal{C}
\end{gathered}
$$

where $\mathbf{n}$ is the normal exterior to the fracture.

Condition (6) is discussed in [17], Sec 2.3. Condition (5) is a simplification of the pressure interface conditions and more careful modeling by upscaling indicates presence of an additional term. We do not dwell on this question here.

We are primarily interested in a fracture propagating due to pressure (water) injection effects. Consequently, we keep the source/sink terms $q$ and set $\sigma_{0}=0$ and neglect gravity effects. 
Remark 1. The main difference in the present work as compared to [17] is that in [17], we solved the $(u, \varphi)$ system monolithically and coupled this system through fixed-stress to the pressure diffraction equation in a sequential procedure. In the present paper, we formulate a fully-coupled system that now allows us to formulate a free energy functional.

The strategy developed in [16]-[17] relies on regularity. For calculations of the displacement and phase field in [16], we required $p \in W^{1,1}\left(0, T ; W^{1,2 r}\left((0, L)^{3}\right)\right)$, with $r>3$. In general, it is not clear that such smoothness can be guaranteed. In [17] we assumed a sharp boundary between the poroelastic domain and the fracture for which $C^{1}$ regularity was needed. The new approach here does not require higher regularity of the phase field. Hence it can be used as a general formulation. The strategy applied in [17] can then be applied to obtain a more accurate approximation of the interface between the poroelastic medium and the fracture.

\subsection{Modeling fractures with a phase-field approach in solid mechanics}

In the following, we present a continuous three-field hydraulic fracturing model. Then we discretize in time and provide a complete mathematical theory for the equations of the corresponding incremental formulation of the system. The mathematical model involves the coupling of a linear elasticity system with a) the pressure equation and b) with an elliptic variational inequality for the phase field variable. With this approach, branching of fractures and heterogeneities in mechanical properties can be effectively treated as demonstrated numerically in Section 7 .

For the elasticity part, our formulation follows Miehe et al. in [12] and enters a generalization of the thermodynamically consistent framework for phase-field models of quasi-static crack propagation in elastic solids, together with incremental variational principles. The phase field equations are based on the variational approach to elastic fractures by Francfort and Marigo ([9] and [4]). Our methodology represents an extension to cracks in a poroelastic medium containing a viscous fluid.

In fracture mechanics, Griffith's criterion states that crack propagation occurs when the elastic energy restitution rate reaches its critical value $G_{c}$. If $\tau$ is the traction force applied at the part of the boundary $\partial_{N} \Omega$, then one can associate to the crack $\mathcal{C}$ the following total energy

$$
E(\mathbf{u}, \mathcal{C})=\int_{\Omega} \frac{1}{2} \mathcal{G} e(\mathbf{u}): e(\mathbf{u}) d x-\int_{\partial_{N} \Omega} \tau \cdot \mathbf{u} d S-\int_{\Omega} F \operatorname{div} \mathbf{u} d x+G_{\mathcal{C}} \mathcal{H}^{2}(\partial \mathcal{C}),
$$

where $F$ is a volume force. In fracture mechanics the crack is considered as a lower dimensional manifold and would correspond to $\partial \mathcal{C}$.

In [9] and 44 a phase field regularization of the term $G_{c} \mathcal{H}^{2}(\partial \mathcal{C})$ was used. The equations in the displacement unknown $\mathbf{u}$ and the phase-field unknown $\varphi$ are derived as the corresponding EulerLagrange equations. They are used for the determination of the cracks spreading in fracture mechanics.

The situation in poroelasticity is more complicated than the classical one from solid mechanics.

\subsection{Specific challenges of phase-field fracture in poroelasticity}

First, we recall that our fracture is a (thin) 3D body and not a surface, with dimensions being small compared to $B$, but large compared to the characteristic pore size $\ell$. At the pore level we have a complex interaction between fracture and the pore skeleton, but such a modeling is presently out of reach. Nevertheless, the experimental observation is that if the stresses (respectively the free energy) cross a threshold, the fracture will start growing and propagating. We will follow the ideas from [9] and [4] and add to the free energy the phase field regularization of the term $G_{\mathcal{C}} \mathcal{H}^{2}(\partial \mathcal{C})$. Since the observed and simulated fractures correspond to smeared two-dimensional surfaces, our treatment is consistent with ideas from the literature (see e.g. [12]). 
Since we do not have a total energy functional for Biot's system (1)-(2), the energy functional (7) must be modified to include pressure effects and a term describing the propagation for some threshold value of the stress. Our strategy is to generalize the notion of the free energy for poroelasticity in order to include the phase-field. This is achieved by regularizing the sharp fracture surface topology in the poroelastic medium by diffusive crack zones described by a scalar auxiliary variable. This variable is a phase-field that interpolates between the unbroken and the broken states of the material. After making all these modifications and taking into the account the pressure field, we will not have an energy functional; however we will derive a generalized free energy. The latter will allow us to construct a Lyapunov functional and as a consequence the incremental problem is well-posed.

\section{Formulation of a fully-coupled displacement, phase-field, pressure system}

In fracture mechanics the phase field unknown $\varphi$ is defined on $B \times(0, T)$ by the regularized crack functional

$$
\Gamma_{\varepsilon}(\varphi)=G_{c} \int_{B}\left(\frac{1}{2 \varepsilon}(1-\varphi)^{2}+\frac{\varepsilon}{2}|\nabla \varphi|^{2}\right) d x=G_{c} \int_{B} \gamma(\varphi, \nabla \varphi) d x
$$

where $\gamma$ is the crack surface density per unit volume and $\varepsilon$ a positive constant. This regularization of $\mathcal{H}^{2}(\partial \mathcal{C})$, in the sense of the $\Gamma$-limit when $\varepsilon \rightarrow 0$, was used in [4]. Even if we do not deal with a surface and its energy, it is observed that the fracture grows in a porous medium if the stress is greater than some critical threshold $G_{c}$. Here, $G_{c}$ depends on the skeleton compressibility and on the fluid viscosity. In classical fracture mechanics setting $G_{c}$ corresponds to the energy release rate. In deference to classical fracture mechanics, the meaning of the problem for $\varepsilon=0$ is not clear and our $\varepsilon$ will be a small positive parameter, but we will not aim to pass to the limit $\varepsilon \rightarrow 0$.

The crack phase-field unknown $\varphi$ is introduced through a regularized elastic energy functional minimization for given pressure field. Then the form of $\Gamma_{\varepsilon}(\varphi)$ yields that meas $\{(x, t) \in B \times(0, T) \mid \varphi(x, t) \leq$ $a<1\} \leq C \varepsilon$. Therefore the parameter $\varepsilon$ represents the characteristic length scale and formally the crack behaves as a surface when $\varepsilon \rightarrow 0$.

Our further considerations are based on the fact that evolution of cracks is fully dissipative in nature. First, the crack phase field $\varphi$ is intuitively a regularization of $1-\chi_{\mathcal{C}}$ and we impose its negative evolution

$$
\partial_{t} \varphi \leq 0
$$

After the seminal work by Biot [3], the Biot stress and the pressure are determined using the strain energy $W$ of a porous elastic medium. $W$ is the free isothermal energy density of the fluid-solid system. After [3], $W$ depends on the strain tensor $e(\mathbf{u})$ and on the increment of the fluid content $\zeta=p / M+\alpha$ div $\mathbf{u}$, i.e. $W=W(e, \zeta)$. Since it is required that

$$
p=\frac{\partial W}{\partial \zeta} \quad \text { and } \quad \sigma=\frac{\partial W}{\partial e},
$$

we have

$$
W(e, \zeta)=\frac{1}{2} \mathcal{G} e(\mathbf{u}): e(\mathbf{u})+p^{2} /(2 M)=\frac{1}{2} \mathcal{G} e(\mathbf{u}): e(\mathbf{u})+\frac{M}{2}(\zeta-\alpha \operatorname{div} \mathbf{u})^{2}
$$


Our idea is to generalize this energy and to use the 'phase field' free energy. It has to take into the account the following effects:

- The degradation of the elastic properties in the fracture, described by the multiplication of the Gassmann tensor $\mathcal{G}$ by the function $A$, such that

$A(\varphi)$ is a smooth monotone increasing function satisfying

$$
A(0)=k>0, k \ll \varepsilon, \quad A^{\prime}(0)=0 \quad \text { and } \quad A(1)=1 .
$$

A usual choice is $A(\varphi)=(1-k) \varphi^{2}+k$.

- The change of the porosity being different in the poroelastic part and in the fracture, and now described through a function $c$ satisfying

$$
\begin{gathered}
c(\varphi) \text { is a smooth bounded function such that } c(0)=c_{F}>0, \\
\inf _{z} c(z)=c_{\min }>0, \quad c^{\prime}(0)=0 \quad \text { and } \quad c(1)=1 / M .
\end{gathered}
$$

Note that the coefficients $A$ and $c$ are naturally defined on $[0,1]$. We extend them for negative values of argument by the value at $\varphi=0$ and pose $A(\varphi)=A\left(\varphi_{+}\right)$and $c(\varphi)=c\left(\varphi_{+}\right)$. Due to constraint (9), $\varphi$ will always remain smaller or equal to 1 . For more discussion we refer to [16] and further comments bellow. Our new 'phase-field' free energy density including fractures is now

$$
W_{\varepsilon}(e, \zeta, \varphi)=\frac{1}{2} A\left(\varphi_{+}\right) \mathcal{G} e(\mathbf{u}): e(\mathbf{u})+\frac{c\left(\varphi_{+}\right) p^{2}}{2}+G_{c}\left(\frac{1}{2 \varepsilon}(1-\varphi)^{2}+\frac{\varepsilon}{2}|\nabla \varphi|^{2}\right) .
$$

We note the presence of the term allowing the fracture to grow, once the threshold energy is crossed. Furthermore the phase field unknown $\varphi$ should allow a smooth change from the fracture zone to the poroelastic zone. For the fracture spreading it is crucial to accurately model the interface and it has to enter the phase field equation on the correct way.

Our strategy is to generalize the well-established approach from fracture mechanics. We proceed in several steps:

\subsection{The elastic part energy functional}

We suppose a given pressure, but bear in mind that this pressure is linked to the displacement and the phase-field unknown. Then we borrow the energy functional from fracture theory of solid mechanics and modify by taking into the account the porosity change. Following modeling of a brittle fracture of fluid-infiltrated material from [7, Sec. 4.4.2., we add a cross term involving the integral of the product between the pressure and the porosity change. This is the main difference with the approaches in the simplified case [15], [16] and [17]. This yields the following functional:

$$
\begin{gathered}
\tilde{\mathcal{E}}_{\varepsilon}(\mathbf{u}, \varphi)=\int_{B} \frac{1}{2} A(\varphi) \mathcal{G} e(\mathbf{u}): e(\mathbf{u}) d x-\int_{\partial_{N} B} \tau \cdot \mathbf{u} d S-\int_{\partial \mathcal{C}} \tau \cdot \mathbf{u} d S \\
-\int_{B} \alpha \varphi p \operatorname{div} \mathbf{u} d x+G_{C} \int_{B}\left(\frac{1}{2 \varepsilon}(1-\varphi)^{2}+\frac{\varepsilon}{2}|\nabla \varphi|^{2}\right) d x \\
+\int_{B} \frac{1}{2}\left(\frac{1}{M}-c\left(\varphi_{+}\right)\right) p^{2} d x
\end{gathered}
$$

The last term is constant with respect to the displacement $\mathbf{u}$ and does not contribute to the displacement equation. 


\subsection{Interface law for the normal stresses on the fracture boundary}

Now we observe that the interface term $\int_{\partial \mathcal{C}} \tau \cdot \mathbf{u} d S \sqrt{12}$ has to be transformed into a volume term. Here, we model the fracture pressure using an interface law. A general description of a crack embedded in a porous medium is illustrated in Figure 1. We concentrate on a simplified situation in which the complex interaction crack/pore structure is neglected. We recall that the crack is a $3 \mathrm{D}$ thin domain with width much smaller than length, then lubrication theory can be applied. Hence, the leading order of the stress in $\mathcal{C}$ is $-p_{f} I$, where $p_{f}$ denotes the fracture pressure. From the poroelastic side the stress is given by $\sigma=\sigma_{0}+\mathcal{G} e(\mathbf{u})-\alpha p_{B} I$, where $p_{B}$ denotes the Biot pressure in the poroelastic medium. At the crack boundary we have the continuity of the contact force, which yields

$$
\sigma \mathbf{n}=\left(\mathcal{G} e(\mathbf{u})-\alpha p_{B} I\right) \mathbf{n}=-p_{f} \mathbf{n},
$$

where $\mathbf{n}$ is the normal vector exterior to the poroelastic domain.

Next, we eliminate the traction crack surface integrals and obtain

$$
\begin{gathered}
\int_{\partial \mathcal{C}} \sigma \mathbf{n w} d S=-\int_{\partial \mathcal{C}} p_{f} w_{n} d S=-\int_{\Omega} \operatorname{div}\left(p_{B} \mathbf{w}\right) d x+\int_{\partial_{N} B} p_{B} w_{n} d S \\
=-\int_{\Omega} p_{B} \operatorname{div} \mathbf{w} d x-\int_{\Omega} \nabla p_{B} \mathbf{w} d x+\int_{\partial_{N} B} p_{B} w_{n} d S,
\end{gathered}
$$

where $w_{n}$ denotes the normal component of the vector function $w$.

Remark 2. In the previous calculations, the first line reads

$$
\int_{\partial \mathcal{C}} \sigma \mathbf{n w} d S=-\int_{\partial \mathcal{C}} p_{f} w_{n, \text { poroelastic }} d S .
$$

Setting now $p=p_{f}$ in the crack and $p=p_{B}$ in the poroelastic medium, then the above calculations yield

$$
\begin{gathered}
-\int_{\partial \mathcal{C}} p_{f} w_{n, \text { poroelastic }} d S=\int_{\mathcal{C}} \nabla p \mathbf{w} d x-\int_{\mathcal{C}} \operatorname{div}(p \mathbf{w}) d x \\
-\int_{\partial \mathcal{C}} p_{f} w_{n, \text { poroelastic }} d S=\int_{\mathcal{C}} \nabla p \mathbf{w} d x-\int_{\partial \mathcal{C}} p\left(w_{n, \text { poroelastic }}-w_{n, \text { crack }}\right) d S .
\end{gathered}
$$

The last term coincides with the virtual work of the pressure force as introduced in [6].

In the above calculations, surface integrals are now treated with Gauss' divergence theorem:

$$
\begin{gathered}
-\int_{\partial_{N} \Omega} \tau \cdot \mathbf{w} d S+\int_{\partial \mathcal{C}} p w_{n} d S-\int_{\Omega} \alpha p \operatorname{div} \mathbf{w} d x=-\int_{\Omega}(\alpha-1) p \operatorname{div} \mathbf{w} d x+ \\
\int_{\Omega} \nabla p \mathbf{w} d x-\int_{\partial_{N} \Omega} \tau \cdot \mathbf{w} d S=-\int_{\Omega}(\alpha-1) p \operatorname{div} \mathbf{w} d x+ \\
\int_{\Omega} \nabla p \cdot \mathbf{w} d x-\int_{\partial_{N} \Omega}\left(\tau \cdot \mathbf{w}+p w_{n}\right) d S .
\end{gathered}
$$

Using (14), $\tilde{\mathcal{E}}_{\varepsilon}$ transforms to

$$
\begin{aligned}
& \hat{\mathcal{E}}_{\varepsilon}(\mathbf{u}, \varphi)=\int_{B} \frac{1}{2} A\left(\varphi_{+}\right) \mathcal{G} e(\mathbf{u}): e(\mathbf{u}) d x-\int_{\partial_{N} B}\left(\tau \cdot \mathbf{u}+p u_{n}\right) d S \\
&+\int_{\Omega}((1-\alpha) p \operatorname{div} \mathbf{u}+\nabla p \cdot \mathbf{u}) d x \\
&+G_{C} \int_{B}\left(\frac{1}{2 \varepsilon}(1-\varphi)^{2}+\frac{\varepsilon}{2}|\nabla \varphi|^{2}\right) d x+\int_{B}\left(\frac{1}{M}-\frac{c\left(\varphi_{+}\right)}{2}\right) p^{2} d x .
\end{aligned}
$$


We suppose that the crack $\mathcal{C}$ does not interact with $\partial_{N} \Omega$. Hence there is no need to insert $\varphi$ in the boundary integral over $\partial_{N} B$.

\subsection{Phase-field formulation of the pressure cross terms}

It remains to extend the pressure cross term

$$
\int_{\Omega}((1-\alpha) p \operatorname{div} \mathbf{u}+\nabla p \cdot \mathbf{u}) d x
$$

to a term with integration over $B$ by adding $\varphi$. Due to the complexity of our model, it is not clear that we will be able to prove the nonnegativity of $\varphi$. Changing the pressure cross term to $\left.\int_{B} \varphi((1-\alpha) p \operatorname{div} \mathbf{u}+\nabla p \cdot \mathbf{u})\right) d x$ is likely to give nonzero values in the domain where $\varphi$ is negative. This is not admissible. Using $\varphi_{+}$instead of $\varphi$ is also unsatisfactory, since the energy functional would not be $C^{1}$ any more. The lack of regularity would lead to complications in numerical simulations. Our approach is to use the function $\varphi_{+}^{2}$ instead of $\varphi$. For $0 \leq \varphi \leq 1$, the choice between $\varphi_{+}^{2}$ and $\varphi_{+}$in the pressure cross term does not affect the phase field approximation. If $\varphi=1-\chi_{\mathcal{C}}$, the two functions coincide.

After the above transformations, we have the following variant of the energy functional (15):

$$
\begin{aligned}
\mathcal{E}_{\varepsilon}(\mathbf{u}, \varphi)= & \int_{B} \frac{1}{2} A\left(\varphi_{+}\right) \mathcal{G} e(\mathbf{u}): e(\mathbf{u}) d x-\int_{\partial_{N} B}\left(\tau \cdot \mathbf{u}+p u_{n}\right) d S \\
& \left.+\int_{B} \varphi_{+}^{2}((1-\alpha) p \operatorname{div} \mathbf{u}+\nabla p \cdot \mathbf{u})\right) d x \\
+G_{c} \int_{B}( & \left.\frac{1}{2 \varepsilon}(1-\varphi)^{2}+\frac{\varepsilon}{2}|\nabla \varphi|^{2}\right) d x+\int_{B}\left(\frac{1}{M}-\frac{c\left(\varphi_{+}\right)}{2}\right) p^{2} d x
\end{aligned}
$$

\subsection{Fréchet derivative of $\mathcal{E}_{\varepsilon}$}

As in the literature, $\{\mathbf{u}, \varphi\}$ are critical points of the non-convex functional $\mathcal{E}_{\varepsilon}$. Taking the Fréchet derivatives yields

$$
\begin{gathered}
-\operatorname{div}\left(\left(A\left(\varphi_{+}\right) \mathcal{G} e(\mathbf{u})\right)+\varphi_{+}^{2} \nabla p+(\alpha-1) \nabla\left(\varphi_{+}^{2} p\right)=0 \text { in } B,\right. \\
\mathcal{L}(\mathbf{u}, \varphi)=-G_{c} \varepsilon \Delta \varphi-\frac{G_{c}}{\varepsilon}(1-\varphi)+\frac{A^{\prime}\left(\varphi_{+}\right)}{2} \mathcal{G} e(\mathbf{u}): e(\mathbf{u})-\frac{c^{\prime}\left(\varphi_{+}\right)}{2} p^{2}+ \\
2(1-\alpha) \varphi_{+} p \operatorname{div} \mathbf{u}+2 \varphi_{+} \nabla p \cdot \mathbf{u} \leq 0 \text { in } B, \\
\partial_{t} \varphi \leq 0 \text { in } B, \\
\partial_{t} \varphi \cdot \mathcal{L}(\mathbf{u}, \varphi)=0 \text { in } B .
\end{gathered}
$$

Here, the last expression (20) is a complementarity condition (namely the Rice condition) linked to the irreversibility constraint (19). 


\subsection{Adding reservoir and fracture pressure equations}

We have seen that under the assumption of the weak compressibility of the fluid, the mass conservation equation reads (2) in the poroelastic part and (4) in the fracture. Following [17] it can be written as the pressure diffraction problem

$$
\theta \partial_{t} p-\operatorname{div}\left(K_{e f f}(\nabla p-\tilde{\mathbf{G}})\right)=\tilde{q} \quad \text { in } \quad B
$$

where

$$
\begin{gathered}
\theta=\theta(x, t)=\chi_{\Omega} \frac{1}{M}+\chi_{\mathcal{C}} c_{F}, \quad \tilde{q}=\tilde{q}(x, t):=\chi_{\Omega} \frac{q_{R}}{\rho_{R}}-\alpha \chi_{\Omega} \partial_{t} \operatorname{div} \mathbf{u}+\chi_{\mathcal{C}} \frac{q_{F}}{\rho_{F}} \\
\tilde{\mathbf{G}}=\chi_{\mathcal{C}} \mathbf{G}, \quad K_{e f f}=\chi_{\Omega} \frac{\mathcal{K}_{R}}{\eta_{R}}+\chi_{\mathcal{C}} \frac{\mathcal{K}_{F}}{\eta_{F}} .
\end{gathered}
$$

We recall that gravity effects are neglected.

The formulation contains the interface between $\Omega$ and $\mathcal{C}$, which changes in time, as the crack propagates. Our idea is to replace the diffraction problem (21) by a phase field equation for the pressure diffraction equation. Following the distinction between terms containing $\alpha-1$ as factor and others from equation (17), we have the following global problem for the pressure:

$$
\begin{gathered}
\partial_{t}\left(c\left(\varphi_{+}\right) p\right)-\operatorname{div}\left(K_{e f f}\left(\varphi_{+}\right)\left(\nabla p-\tilde{\mathbf{G}}\left(\varphi_{+}\right)\right)\right)+(\alpha-1) \partial_{t}\left(\varphi_{+}^{2} \operatorname{div} \mathbf{u}\right)+ \\
\operatorname{div}\left(\partial_{t}\left(\varphi_{+}^{2} \mathbf{u}\right)\right)=\tilde{q} \text { in } B .
\end{gathered}
$$

Note that for $\varphi=0$ and $\varphi=1$ we have again equation $(21)$. $\tilde{\mathbf{G}}(0)=\mathbf{G}$ and $\tilde{\mathbf{G}}(1)=0$.

\subsection{The fully coupled system for $\{\mathbf{u}, \varphi, p\}$}

We now summarize the Euler-Lagrange equations from STEP 4 and the phase-field pressure diffraction problem from STEP 5 and obtain the following fully-coupled system: Find $\mathbf{u}, \varphi, p$ such that:

$$
\begin{gathered}
-\operatorname{div}\left(A\left(\varphi_{+}\right) \mathcal{G} e(\mathbf{u})\right)+\varphi_{+}^{2} \nabla p+(\alpha-1) \nabla\left(\varphi_{+}^{2} p\right)=0 \quad \text { in } B, \\
\mathbf{u}=0 \quad \text { and } \quad-K_{\text {eff }}\left(\varphi_{+}\right)\left(\nabla p-\tilde{\mathbf{G}}\left(\varphi_{+}\right)\right) \cdot \mathbf{n}=v^{i n j} \text { on } \quad \partial_{D} B, \\
-\mathcal{G} e(\mathbf{u}) \mathbf{n}=\tau+p^{b d r y} \mathbf{n} \quad \text { on } \quad \partial_{N} B,
\end{gathered}
$$




$$
\begin{gathered}
-G_{c} \varepsilon \Delta \varphi-\frac{G_{c}}{\varepsilon}(1-\varphi)+\frac{1}{2} A^{\prime}\left(\varphi_{+}\right) \mathcal{G} e(\mathbf{u}): e(\mathbf{u})-\frac{1}{2} c^{\prime}\left(\varphi_{+}\right) p^{2} \\
+2 \varphi_{+}(((1-\alpha) p \operatorname{div} \mathbf{u}+\nabla p \cdot \mathbf{u}) \leq 0 \quad \text { in } B \\
\partial_{t} \varphi \leq 0 \quad \text { on } \quad B \\
\left(-G_{c} \varepsilon \Delta \varphi-\frac{G_{c}}{\varepsilon}(1-\varphi)+\frac{1}{2} A^{\prime}\left(\varphi_{+}\right) \mathcal{G} e(\mathbf{u}): e(\mathbf{u})-\frac{1}{2} c^{\prime}\left(\varphi_{+}\right) p^{2}\right. \\
+2 \varphi_{+}(((1-\alpha) p \operatorname{div} \mathbf{u}+\nabla p \cdot \mathbf{u})) \partial_{t} \varphi=0 \text { in } B \\
\varphi(x, 0)=\varphi^{0}(x) \quad \text { and } \quad \frac{\partial \varphi}{\partial \mathbf{n}}=0 \quad \text { on } \partial B
\end{gathered}
$$

where (24) and (31) contain the boundary conditions for the displacement and the pressure.

\subsection{Weak formulation of the fully coupled system}

As functional spaces of admissible displacements and pressures, we choose

$$
\begin{aligned}
& V_{U}=\left\{\mathbf{z} \in H^{1}(B)^{3} \mid \mathbf{z}=0 \text { on } \partial_{D} B\right\} \\
& V_{P}=\left\{\pi \in H^{1}(B) \mid \pi=0 \text { on } \partial_{N} B\right\} .
\end{aligned}
$$

We proceed by writing the variational formulation corresponding to problem (23)-(31):

Find $\{\mathbf{u}, \varphi, p\}$ such that

$$
\begin{gathered}
\int_{B} A\left(\varphi_{+}\right) \mathcal{G} e(\mathbf{u}): e(\mathbf{w}) d x+\int_{B} \varphi_{+}^{2} \nabla p \cdot \mathbf{w} d x+(\alpha-1) \int_{B} \nabla\left(\varphi_{+}^{2} p\right) \cdot \mathbf{w} d x \\
=\int_{\partial_{N} B}\left(\tau \cdot \mathbf{w}+p^{b d r y} w_{n}\right) d S, \quad \forall \mathbf{w} \in V_{U} \quad \text { a.e. on }(0, T) \\
\mathcal{B}(\varphi, \psi)=G_{c} \int_{B}\left(\varepsilon \nabla \varphi \cdot \nabla \psi-\frac{1}{\varepsilon}(1-\varphi) \psi\right) d x+\frac{1}{2} \int_{B}\left(A^{\prime}\left(\varphi_{+}\right) \mathcal{G} e(\mathbf{u}): e(\mathbf{u})\right. \\
\left.-c^{\prime}\left(\varphi_{+}\right) p^{2}\right) \psi d x+2 \int_{B} \varphi_{+}(((1-\alpha) p \operatorname{div} \mathbf{u}+\nabla p \cdot \mathbf{u}) \psi d x \leq 0 \quad \text { a.e. on }(0, T), \\
\forall \psi \in H^{1}(B), \quad \psi \geq 0 \text { a.e. on } B
\end{gathered}
$$




$$
\begin{gathered}
\partial_{t} \varphi \leq 0 \quad \text { a.e. on } B \times(0, T) ; \\
\mathcal{B}\left(\varphi, \partial_{t} \varphi\right)=0 \quad \text { a.e. on }(0, T) ; \\
\varphi(x, 0)=\varphi^{0}(x) \quad \text { on } \quad B, \quad 0 \leq \varphi^{0}(x) \leq 1 ;
\end{gathered}
$$

$$
\begin{gathered}
\frac{d}{d t} \int_{B} c\left(\varphi_{+}\right) p z d x+\int_{B}\left(K_{e f f}\left(\varphi_{+}\right)\left(\nabla p-\tilde{\mathbf{G}}\left(\varphi_{+}\right)\right)\right) \cdot \nabla z d x+ \\
(\alpha-1) \int_{B} \partial_{t}\left(\varphi_{+}^{2} \operatorname{div} \mathbf{u}\right) z d x+\int_{B} \operatorname{div}\left(\partial_{t}\left(\varphi_{+}^{2} \mathbf{u}\right)\right) z d x=\int_{B} \tilde{q} z d x \\
-\int_{\partial_{D} B} v^{i n j} z d S, \quad \forall z \in V_{P} \quad \text { a.e. on }(0, T) ; \\
p(x, 0)=p^{0}(x) \quad \text { on } \quad B ; \quad p=p^{b d r y} \quad \text { on } \quad \partial_{N} B .
\end{gathered}
$$

We notice that $\mathcal{B}\left(\varphi, \partial_{t} \varphi\right)=0$ is just another notation for the complementarity condition.

We will undertake the study of the incremental (i.e. the time discretized problem) in Sec. 4 . Nevertheless, the basic idea of this phase-field formulation is to formulate a Lyapunov functional for our full model that generalizes the free energy. The corresponding calculations follow in the next step.

\subsection{Construction of the Lyapunov functional}

Proposition 1. Let us suppose smooth data and a smooth solution $\{\mathbf{u}, \varphi, p\}$ for problem (32)-(38). Then the following energy equality holds

$$
\begin{gathered}
\frac{1}{2} \frac{d}{d t}\left\{\int _ { B } \left(A\left(\varphi_{+}\right) \mathcal{G} e(\mathbf{u}): e(\mathbf{u})+G_{c}\left(\varepsilon|\nabla \varphi|^{2}+\frac{1}{\varepsilon}(1-\varphi)^{2}\right)+c\left(\varphi_{+}\right) p^{2}-\right.\right. \\
\left.2 c\left(\varphi_{+}\right) p p^{b d r y}-2(\alpha-1) \varphi_{+}^{2} \operatorname{div} \mathbf{u} p^{b d r y}-2 \operatorname{div}\left(\varphi_{+}^{2} \mathbf{u}\right) p^{b d r y}\right) d x \\
\left.-2 \int_{\partial_{N} B}\left(\tau \cdot \mathbf{u}+(1-\alpha) p^{b d r y} u_{n}\right) d S\right\}+\int_{B} K_{e f f}\left(\varphi_{+}\right)|\nabla p|^{2} d x= \\
-\int_{\partial_{N} B}\left(\partial_{t} \tau \cdot \mathbf{u}+\partial_{t} p^{b d r y} u_{n}\right) d S-\int_{B} K_{e f f}\left(\varphi_{+}\right)\left(\nabla p-\tilde{\mathbf{G}}\left(\varphi_{+}\right)\right) \cdot \nabla p^{b d r y} d x \\
-\int_{B} c\left(\varphi_{+}\right) p \partial_{t} p^{b d r y} d x-(\alpha-1) \int_{B} \varphi_{+}^{2} \operatorname{div} \mathbf{u} \partial_{t} p^{b d r y} d x \\
-\int_{B} \operatorname{div}\left(\varphi_{+}^{2} \mathbf{u}\right) \partial_{t} p^{b d r y} d x+\int_{B} K_{e f f}\left(\varphi_{+}\right) \tilde{\mathbf{G}}\left(\varphi_{+}\right) \cdot \nabla p^{b d r y} d x \\
-\int_{\partial_{D} B} v^{i n j}\left(p-p^{b d r y}\right) d S+\int_{B} \tilde{q}\left(p-p^{b d r y}\right) d x .
\end{gathered}
$$

Proof. We will discuss the proof only for the incremental problem, in Theorem 2.

Remark 3. The free energy for problem (32)-(38) is

$$
\{\mathbf{u}, \varphi, p\} \rightarrow \frac{1}{2}\left(A\left(\varphi_{+}\right) \mathcal{G} e(\mathbf{u}): e(\mathbf{u})+G_{c}\left(\varepsilon|\nabla \varphi|^{2}+\frac{1}{\varepsilon}(1-\varphi)^{2}\right)+c\left(\varphi_{+}\right) p^{2}\right) .
$$




\section{An incremental formulation of the fully-coupled system}

In the following, we consider a quasi-static formulation where velocity changes are small. First, we derive an incremental form, i.e., we replace the time derivative in inequality (9) with a discretized version; more precisely

$$
\partial_{t} \varphi \rightarrow \partial_{\Delta t} \varphi=(\varphi-\Phi) /(\Delta t)
$$

where $\Delta t>0$ is the time step and $\Phi$ is the phase field from the previous time step. After time discretization, our quasistatic constrained minimization problem becomes a stationary problem, called the incremental problem. The entropy condition $(9)$ is imposed in its discretized form and we introduce a convex set $K$ :

$$
K=\left\{\psi \in H^{1}(B) \mid \psi \leq \Phi \leq 1 \text { a.e. on } B\right\} .
$$

Note that the value of the phase field unknown $\varphi$ from the previous time step enters only the convex set $K$, as the obstacle $\Phi$. $U$ and $P$ will denote the values of the displacement and the pressure from the previous time step.

Let us write the incremental form of problem (23)-(31).

$$
\begin{gathered}
-\operatorname{div}\left(A\left(\varphi_{+}\right) \mathcal{G} e(\mathbf{u})\right)+\varphi_{+}^{2} \nabla p+(\alpha-1) \nabla\left(\varphi_{+}^{2} p\right)=0 \quad \text { in } B, \\
\mathbf{u}=0 \quad \text { and } \quad-K_{e f f}(1)\left(\nabla p-\tilde{\mathbf{G}}\left(\Phi_{+}\right)\right) \cdot \mathbf{n}=v^{i n j} \text { on } \partial_{D} B, \\
\mathcal{G} e(\mathbf{u}) \mathbf{n}=\tau+p^{b d r y} \mathbf{n} \quad \text { on } \quad \partial_{N} B,
\end{gathered}
$$

$$
\begin{gathered}
-G_{c} \varepsilon \Delta \varphi-\frac{G_{c}}{\varepsilon}(1-\varphi)+\frac{1}{2} \mathcal{A}(\varphi) \mathcal{G} e(\mathbf{U}): e(\mathbf{U})-\frac{1}{2} \mathcal{J}(\varphi) P(2 p-P) \\
+\mathcal{D}(\varphi)((1-\alpha) p \operatorname{div} \mathbf{U}+\nabla p \cdot \mathbf{U}) \leq 0 \quad \text { in } B \\
\partial_{\Delta t} \varphi \leq 0 \quad \text { on } \quad B \\
\left(-G_{c} \varepsilon \Delta \varphi-\frac{G_{c}}{\varepsilon}(1-\varphi)+\frac{1}{2} \mathcal{A}(\varphi) \mathcal{G} e(\mathbf{U}): e(\mathbf{U})+\frac{1}{2} \mathcal{J}(\varphi) P(2 p-P)\right. \\
+\mathcal{D}(\varphi)(((1-\alpha) p \operatorname{div} \mathbf{U}+\nabla p \cdot \mathbf{U})) \partial_{\Delta t} \varphi=0 \text { in } B \\
\varphi(x, 0)=\varphi^{0}(x) \quad \text { and } \quad \frac{\partial \varphi}{\partial \mathbf{n}}=0 \quad \text { on } \partial B ;
\end{gathered}
$$

$$
\begin{aligned}
& \partial_{\Delta t}\left(c\left(\varphi_{+}\right) p\right)-\operatorname{div}(\left.K_{e f f}\left(\Phi_{+}\right)\left(\nabla p-\tilde{\mathbf{G}}\left(\Phi_{+}\right)\right)\right)+(\alpha-1) \partial_{\Delta t}\left(\varphi_{+}^{2} \operatorname{div} \mathbf{u}\right) \\
&+\operatorname{div}\left(\partial_{\Delta t}\left(\varphi_{+}^{2} \mathbf{u}\right)\right)=\tilde{q} \text { in } B \\
& \varphi(x, 0)=\varphi^{0}(x) \text { and } p(x, 0)=p^{0}(x) \text { on } B ; \quad p=p^{b d r y} \text { on } \partial_{N} B
\end{aligned}
$$


where (47) is the strong form of Rice' condition. The functions $\mathcal{A}, \mathcal{J}$ and $\mathcal{D}$ correspond to discrete derivatives of $A, c$ and $\varphi^{2}$ and are given by

$$
\mathcal{A}(\varphi)=\frac{A\left(\varphi_{+}\right)-A\left(\Phi_{+}\right)}{\varphi-\Phi} ; \quad \mathcal{J}(\varphi)=\frac{c\left(\varphi_{+}\right)-c\left(\Phi_{+}\right)}{\varphi-\Phi} \quad \text { and } \quad \mathcal{D}(\varphi)=\frac{\varphi_{+}^{2}-\Phi_{+}^{2}}{\varphi-\Phi}
$$

Here, the system (42)-(47) is a variational inequality; in [12] a penalization term is used for solving the inequality. To the best of our knowledge the modeling of the full system and its analysis are new in the literature.

\section{Well-posedness of the model}

We start by making the following assumptions on the data

\section{Hypothesis 1.}

$\mathcal{G}$ is a positive definite constant rank-4 tensor,

$\tilde{\mathbf{G}}$ is a bounded continuous function of $\varphi_{+}, x$ and $t$,

$K_{\text {eff }}$ is a continuous bounded symmetric matrix, positively definite,

with the ellipticity constant independent of the argument,

$A, c$ are continuously differentiable functions,

taking values between two positive constants;

$\Phi \in H^{1}(B), \quad \Phi \leq 1 \quad$ a.e. on $\left.B ; \quad \tau \in L^{2}\left(\partial_{N} B\right)\right)$,

$p^{b d r y} \in H^{1}(B), \quad v^{i n j} \in L^{2}\left(\partial_{D} B\right) \quad$ and $\quad \tilde{q} \in L^{2}(B)$.

\subsection{A finite dimensional approximation}

Let $\left\{\psi_{r}\right\}_{r \in \mathbb{N}}$ be a smooth basis for $H^{1}(B),\left\{\pi_{r}\right\}_{r \in \mathbb{N}}$ a smooth basis for $V_{P}$ and $\left\{\mathbf{w}^{r}\right\}_{r \in \mathbb{N}}$ be a smooth basis for $V_{U}$. We start by defining a finite dimensional approximation to the problem (42)-(50).

Definition 1 (of a penalized approximation). Let us suppose that the data satisfy Hypothesis 1 . a penalization parameter $\delta \in \mathbb{R}$ and in particular, let $\delta:=M \in \mathbb{N}$ in this section. Let $\tilde{\varphi}=\inf \left\{1, \varphi_{+}\right\}$ and let $V_{U}^{M}=\operatorname{span}\left\{\mathbf{w}^{r}\right\}_{r=1, \ldots, M}, V_{P}^{M}=\operatorname{span}\left\{\pi_{r}\right\}_{r=1, \ldots, M}$ and $W^{M}=\operatorname{span}\left\{\psi_{r}\right\}_{r=1, \ldots, M}$. The triple $\left\{\mathbf{u}^{M}, \varphi^{M}, p^{M}\right\}, \mathbf{u}^{M}=\sum_{r=1}^{M} a_{r} \mathbf{w}^{r}, p^{M}=p^{b d r y}+\sum_{r=1}^{M} d_{r} \pi_{r}$ and $\varphi^{M}=\Phi+\sum_{r=1}^{M} b_{r} \psi_{r}$, is a finite dimensional approximative solution for problem (42)-(50) if it satisfies the discrete variational 
formulation

$$
\begin{gathered}
\int_{B}\left(A\left(\tilde{\varphi}^{M}\right) \mathcal{G} e\left(\mathbf{u}^{M}\right): e\left(\mathbf{w}^{r}\right)+\left(\left(\tilde{\varphi}^{M}\right)^{2} \nabla p^{M}+(\alpha-1) \nabla\left(\left(\tilde{\varphi}^{M}\right)^{2} p^{M}\right)\right) \cdot \mathbf{w}^{r}\right) d x \\
=\int_{\partial_{N} B}\left(\tau \cdot \mathbf{w}^{r}+p^{b d r y} w_{n}^{r}\right) d S, \quad \forall r=1, \ldots, M
\end{gathered}
$$

$$
\begin{gathered}
G_{c} \int_{B}\left(-\frac{1}{\varepsilon}\left(1-\varphi^{M}\right) \psi_{r}+\varepsilon \nabla \varphi^{M} \cdot \nabla \psi_{r}\right) d x+\int_{B} \delta\left(\varphi^{M}-\Phi\right)_{+} \psi_{r} d x \\
+\int_{B} \mathcal{D}\left(\tilde{\varphi}^{M}\right)\left(\left((1-\alpha) p^{M} d i v \mathbf{U}+\nabla p^{M} \cdot \mathbf{U}\right) \psi_{r} d x+\right. \\
\frac{1}{2} \int_{B}\left(\mathcal{A}\left(\tilde{\varphi}^{M}\right) \mathcal{G} e(\mathbf{U}): e(\mathbf{U})-\mathcal{J}\left(\tilde{\varphi}^{M}\right) P\left(2 p^{M}-P\right)\right) \psi_{r} d x=0, \quad \forall r=1, \ldots, M,
\end{gathered}
$$

$$
\begin{gathered}
\int_{B}\left(c\left(\tilde{\varphi}^{M}\right) p^{M}+\operatorname{div}\left(\left(\tilde{\varphi}^{M}\right)^{2} \mathbf{u}^{M}\right)+(\alpha-1)\left(\tilde{\varphi}^{M}\right)^{2} \operatorname{div} \mathbf{u}^{M}\right) \pi_{r} d x \\
+\Delta t \int_{B}\left(K_{e f f}\left(\Phi_{+}\right)\left(\nabla p^{M}-\tilde{\mathbf{G}}\left(\Phi_{+}\right)\right)\right) \cdot \nabla \pi_{r} d x=\Delta t \int_{B} \tilde{q} \pi_{r} d x \\
-\Delta t \int_{\partial_{D} B} v^{i n j} \pi_{r} d S+\int_{B}\left(c\left(\Phi_{+}\right) P+\operatorname{div}\left(\Phi_{+}^{2} \mathbf{U}\right)+\right. \\
\left.(\alpha-1) \Phi_{+}^{2} \operatorname{div} \mathbf{U}\right) \pi_{r} d x, \quad \forall r=1, \ldots, M,
\end{gathered}
$$

with

$$
\begin{gathered}
\mathcal{A}\left(\tilde{\varphi}^{n}\right)=\frac{A\left(\tilde{\varphi}^{n}\right)-A\left(\Phi_{+}\right)}{\varphi^{n}-\Phi} ; \quad \mathcal{J}\left(\tilde{\varphi}^{n}\right)=\frac{c\left(\tilde{\varphi}^{n}\right)-c\left(\Phi_{+}\right)}{\varphi^{n}-\Phi} \\
\text { and } \quad \mathcal{D}\left(\tilde{\varphi}^{n}\right)=\frac{\left(\tilde{\varphi}^{n}\right)^{2}-\Phi_{+}^{2}}{\varphi^{n}-\Phi}, \quad n=1, \ldots, M .
\end{gathered}
$$

Proposition 2. We suppose Hypothesis 1. Then there exists a finite dimensional penalized approximative solution for problem (52)-(54) that satisfies the a priori estimate

$$
\begin{gathered}
G_{c} \int_{B}\left(\frac{\left(\varphi^{M}\right)^{2}}{\varepsilon}+\varepsilon\left|\nabla \varphi^{M}\right|^{2}\right) d x+\int_{B} \delta\left(\varphi^{M}-\Phi\right)_{+}^{2} d x+\left\|\mathbf{u}^{M}\right\|_{H^{1}(B)^{3}}^{2} \\
+\left\|p^{M}\right\|_{H^{1}(B)}^{2} \leq C,
\end{gathered}
$$

where $C$ is independent of $M$ and $\delta$. 
Proof. (of Proposition 2) Let $\xi=\left\{a_{r}, b_{r}, d_{r}\right\}_{r=1, \ldots, M}=\left\{\xi^{1}, \xi^{2}, \xi^{3}\right\}$ and $X$ the finite dimensional space spanned by the set of all such $\xi$. $X$ is isomorphic to $\mathbb{R}^{3 M}$ and we take the natural scalar product. After setting

$$
\begin{gathered}
P_{1, r}(\xi)=\int_{B}\left(A\left(\tilde{\varphi}^{M}\right) \mathcal{G} e\left(\mathbf{u}^{M}\right): e\left(\mathbf{w}^{r}\right)+(\alpha-1) \nabla\left(\left(\tilde{\varphi}^{M}\right)^{2} p^{M}\right) \cdot \mathbf{w}^{r}\right) d x+ \\
\int_{B}\left(\tilde{\varphi}^{M}\right)^{2} \nabla p^{M} \cdot \mathbf{w}^{r} d x-\int_{\partial_{N} B}\left(\tau \cdot \mathbf{w}^{r}+p^{b d r y} w_{n}^{r}\right) d S, \quad r=1, \ldots, M
\end{gathered}
$$

$$
\begin{gathered}
P_{2, r}(\xi)=G_{c} \int_{B}\left(-\frac{1}{\varepsilon}\left(1-\varphi^{M}\right) \psi_{r}+\varepsilon \nabla \varphi^{M} \cdot \nabla \psi_{r}\right) d x+ \\
\int_{B} \psi_{r} \delta\left(\varphi^{M}-\Phi\right)_{+} d x+\int_{B} \mathcal{D}\left(\tilde{\varphi}^{M}\right)\left(\left((1-\alpha) p^{M} \operatorname{div} \mathbf{U}+\nabla p^{M} \cdot \mathbf{U}\right) \psi_{r} d x\right. \\
+\frac{1}{2} \int_{B}\left(\mathcal{A}\left(\tilde{\varphi}^{M}\right) \mathcal{G} e(\mathbf{U}): e(\mathbf{U})-\mathcal{J}\left(\tilde{\varphi}^{M}\right) P\left(2 p^{M}-P\right)\right) \psi_{r} d x, \quad \forall r=1, \ldots, M,
\end{gathered}
$$

$$
\begin{gathered}
P_{3, r}(\xi)=\int_{B}\left(c\left(\tilde{\varphi}^{M}\right) p^{M}+\operatorname{div}\left(\left(\tilde{\varphi}^{M}\right)^{2} \mathbf{u}^{M}\right)+(\alpha-1)\left(\tilde{\varphi}^{M}\right)^{2} \operatorname{div} \mathbf{u}^{M}\right) \pi_{r} d x \\
+\Delta t \int_{B}\left(K_{e f f}\left(\Phi_{+}\right)\left(\nabla p^{M}-\tilde{\mathbf{G}}\left(\Phi_{+}\right)\right)\right) \cdot \nabla \pi_{r} d x-\Delta t \int_{B} \tilde{q} \pi_{r} d x+ \\
\Delta t \int_{\partial_{D} B} v^{i n j} \pi_{r} d S+\int_{B}\left(c\left(\Phi_{+}\right) P+\operatorname{div}\left(\Phi_{+}^{2} \mathbf{U}\right)+\right. \\
\left.(\alpha-1) \Phi_{+}^{2} \operatorname{div} \mathbf{U}\right) \pi_{r} d x, \quad \forall r=1, \ldots, M,
\end{gathered}
$$

we see that problem (52)-(54) has a solution if and only if equation $\mathbf{P}(\xi)=0$ has a solution.

The nonlinear mapping $\mathbf{P}$ is obviously continuous between $X$ and $X$. Using a well-known corollary ${ }^{1}$ of Brouwer's fixed point theorem, it is enough to prove that $(\mathbf{P}(\xi), \xi)_{X}>0$ for $\xi$ with sufficiently large norm. Existence of at least one root would follow.

We start by multiplying $P_{1, r}(\xi)$ by $a_{r}$ and taking the sum with respect to $r$. It yields

$$
\begin{aligned}
\left(P_{1}(\xi), \xi^{1}\right) & =\int_{B}\left(A\left(\tilde{\varphi}^{M}\right) \mathcal{G} e\left(\mathbf{u}^{M}\right): e\left(\mathbf{u}^{M}\right)+(\alpha-1) \nabla\left(\left(\tilde{\varphi}^{M}\right)^{2} p^{M}\right) \cdot \mathbf{u}^{M}\right) d x \\
& +\int_{B}\left(\tilde{\varphi}^{M}\right)^{2} \nabla p^{M} \cdot \mathbf{u}^{M} d x-\int_{\partial_{N} B}\left(\tau \cdot \mathbf{u}^{M}+p^{b d r y} u_{n}^{M}\right) d S
\end{aligned}
$$

Since $\mathbf{u}^{M} \in V_{U}$, the trace theorem and Korn's inequality yield

$$
\begin{gathered}
\left|\int_{\partial_{N} B}\left(\tau \cdot \mathbf{u}^{M}+p^{b d r y} u_{n}^{M}\right) d S\right| \leq C\left\|e\left(\mathbf{u}^{M}\right)\right\|_{L^{2}(B)}\left(\|\tau\|_{L^{2}\left(\partial_{N} B\right)^{3}}\right. \\
\left.+\left\|p^{b d r y}\right\|_{L^{2}\left(\partial_{N} B\right)}\right) \\
A\left(\tilde{\varphi}^{M}\right) \mathcal{G} e\left(\mathbf{u}^{M}\right): e\left(\mathbf{u}^{M}\right) \geq C_{K}\left\|\mathbf{u}^{M}\right\|_{H^{1}(B)} .
\end{gathered}
$$

\footnotetext{
${ }^{1}$ Lemma 1.4. (see e.g. R. Temam, Navier-Stokes Equations, page 164) Let $X$ be a finite dimensional Hilbert space with scalar product $(\cdot, \cdot)_{X}$ and norm $\|\cdot\|_{X}$ and let $\mathbf{P}$ be a continuous from $X$ into itself such that

$$
(\mathbf{P}(\xi), \xi)_{X}>0 \text { for }\|\xi\|_{X}=R>0 .
$$

Then there exists $\xi \in X,\|\xi\|_{X} \leq R$, such that $\mathbf{P}(\xi)=0$.
} 
After inserting 61)-62 into 60), we get

$$
\begin{gathered}
\left(P_{1}(\xi), \xi^{1}\right) \geq C\left\|\mathbf{u}^{M}\right\|_{H^{1}(B)}^{2}-C \\
+\int_{B}(\alpha-1) \nabla\left(\left(\tilde{\varphi}^{M}\right)^{2} p^{M}\right) \cdot \mathbf{u}^{M} d x+\int_{B}\left(\tilde{\varphi}^{M}\right)^{2} \nabla p^{M} \cdot \mathbf{u}^{M} d x .
\end{gathered}
$$

Next we have

$$
\begin{gathered}
\left(P_{2}(\xi), \xi^{2}\right)=G_{c} \int_{B}\left(-\frac{1}{\varepsilon}\left(1-\varphi^{M}\right)\left(\varphi^{M}-\Phi\right)+\varepsilon \nabla \varphi^{M} \cdot \nabla\left(\varphi^{M}-\Phi\right)\right) d x+ \\
\int_{B} \delta\left(\varphi^{M}-\Phi\right)_{+}^{2} d x+\int_{B} \mathcal{D}\left(\tilde{\varphi}^{M}\right)\left(\left((1-\alpha) p^{M} \operatorname{div} \mathbf{U}+\nabla p^{M} \cdot \mathbf{U}\right)\left(\varphi^{M}-\Phi\right) d x\right. \\
+\frac{1}{2} \int_{B}\left(\mathcal{A}\left(\tilde{\varphi}^{M}\right) \mathcal{G} e(\mathbf{U}): e(\mathbf{U})-\mathcal{J}\left(\tilde{\varphi}^{M}\right) P\left(2 p^{M}-P\right)\right)\left(\varphi^{M}-\Phi\right) d x
\end{gathered}
$$

Estimating different the terms is straightforward:

$$
\begin{aligned}
& G_{c} \int_{B}-\frac{1}{\varepsilon}\left(1-\varphi^{M}\right)\left(\varphi^{M}-\Phi\right) d x \geq-\frac{C}{\varepsilon}+G_{c} \int_{B} \frac{\left(\varphi^{M}\right)^{2}}{2 \varepsilon} d x \\
& -\frac{G_{c}}{\varepsilon}\|\Phi\|_{L^{2}(B)} ; \\
& \left|\int_{B} \mathcal{J}\left(\tilde{\varphi}^{M}\right) P\left(2 p^{M}-P\right)\left(\varphi^{M}-\Phi\right) d x\right| \leq C\|P\|_{L^{2}(B)}\left(\|P\|_{L^{2}(B)}\right. \\
& \left.+\left\|p^{M}\right\|_{L^{2}(B)}\right) ; \\
& \left|\int_{B} \mathcal{D}\left(\tilde{\varphi}^{M}\right) \nabla p^{M} \cdot \mathbf{U}\left(\varphi^{M}-\Phi\right) d x\right| \leq C|| \mathbf{U}\left\|_{L^{2}(B)}|| \nabla p^{M}\right\|_{L^{2}(B)} ; \\
& \left|\int_{B} \mathcal{D}\left(\tilde{\varphi}^{M}\right) p^{M} \operatorname{div} \mathbf{U}\left(\varphi^{M}-\Phi\right) d x\right| \leq C|| \mathbf{U}\left\|_{H^{1}(B)}|| p^{M}\right\|_{L^{2}(B)} .
\end{aligned}
$$

Therefore, we have

$$
\begin{gathered}
\left(P_{2}(\xi), \xi^{2}\right) \geq G_{c} \int_{B}\left(\frac{\left(\varphi^{M}\right)^{2}}{2 \varepsilon}+\varepsilon\left|\nabla \varphi^{M}\right|^{2}\right) d x-C\|P\|_{L^{2}(B)}\left(\|P\|_{L^{2}(B)}\right. \\
\left.+\left\|p^{M}\right\|_{L^{2}(B)}\right)-C\|\mathbf{U}\|_{H^{1}(B)^{3}}\left\|p^{M}\right\|_{H^{1}(B)^{3}}-C-C \delta\left\|\varphi^{M}\right\|_{L^{1}(B)} \\
-C\|\Phi\|_{H^{1}(B)^{3}}+\delta \int_{B}\left(\varphi^{M}-\Phi\right)_{+}^{2} d x .
\end{gathered}
$$

It remains to consider the third component $P_{3}$ of $P$ :

$$
\begin{gathered}
\left(P_{3}(\xi), \xi^{3}\right)=\int_{B}\left(c\left(\tilde{\varphi}^{M}\right) p^{M}+\operatorname{div}\left(\left(\tilde{\varphi}^{M}\right)^{2} \mathbf{u}^{M}\right)+(\alpha-1)\left(\tilde{\varphi}^{M}\right)^{2} \operatorname{div} \mathbf{u}^{M}\right)\left(p^{M}\right. \\
\left.-p^{b d r y}\right) d x+\Delta t \int_{B} K_{e f f}\left(\tilde{\varphi}_{p}\right)\left(\nabla p^{M}-\tilde{\mathbf{G}}\left(\Phi_{+}\right)\right) \cdot \nabla\left(p^{M}-p^{b d r y}\right) d x \\
-\Delta t \int_{\partial_{D} B} v^{i n j}\left(p^{M}-p^{b d r y}\right) d S-\Delta t \int_{B} \tilde{q}\left(p^{M}-p^{b d r y}\right) d x+ \\
\int_{B}\left(c\left(\Phi_{+}\right) P+\operatorname{div}\left(\Phi_{+}^{2} \mathbf{U}\right)+(\alpha-1) \Phi_{+}^{2} \operatorname{div} \mathbf{U}\right)\left(p^{M}-p^{b d r y}\right) d x
\end{gathered}
$$

The idea is to put (63), (66) and (67) together and to obtain a convenient lower bound. A quick inspection of the obtained estimates shows that it remains to control the cross terms in $\left(P_{1}(\xi), \xi^{1}\right)$ 
and in $\left(P_{3}(\xi), \xi^{3}\right)$. Putting them together yields

$$
\begin{gathered}
\int_{B}(\alpha-1)\left(\nabla\left(\left(\tilde{\varphi}^{M}\right)^{2}\left(p^{M}-p^{b d r y}\right)\right) \cdot \mathbf{u}^{M}+\left(\tilde{\varphi}^{M}\right)^{2} \operatorname{div} \mathbf{u}^{M}\left(p^{M}-p^{b d r y}\right)\right) d x= \\
(\alpha-1) \int_{B} \operatorname{div}\left(\left(\tilde{\varphi}^{M}\right)^{2}\left(p^{M}-p^{b d r y}\right) \mathbf{u}^{M}\right) d x=0 \\
\int_{B}\left(\left(\tilde{\varphi}^{M}\right)^{2} \nabla\left(p^{M}-p^{b d r y}\right) \cdot \mathbf{u}^{M}+\operatorname{div}\left(\left(\tilde{\varphi}^{M}\right)^{2} \mathbf{u}^{M}\right)\left(p^{M}-p^{b d r y}\right)\right) d x=0
\end{gathered}
$$

Therefore we have

$$
\begin{gathered}
\left.(\mathbf{P}(\xi), \xi)=\left(P_{1}(\xi), \xi^{1}\right)+\left(P_{2}(\xi), \xi^{2}\right)+P_{3}(\xi), \xi^{3}\right) \geq G_{C} \int_{B}\left(\frac{\left(\varphi^{M}\right)^{2}}{4 \varepsilon}+\varepsilon\left|\nabla \varphi^{M}\right|^{2}\right) d x \\
+C\left\|\mathbf{u}^{M}\right\|_{H^{1}(B)^{3}}^{2}+C\left\|p^{M}\right\|_{H^{1}(B)}^{2}-C\|P\|_{H^{1}(B)}^{2}-C\|\mathbf{U}\|_{H^{1}(B)^{3}}^{2}-C \\
-C\|\Phi\|_{H^{1}(B)^{3}}+\delta \int_{B}\left(\varphi^{M}-\Phi\right)_{+}^{2} d x
\end{gathered}
$$

It follows from 68 that $(\mathbf{P}(\xi), \xi)>0$ for $\|\xi\|=R$, with sufficiently large $R$.

Obviously corresponding solutions $\left\{\mathbf{u}^{M}, \varphi^{M}, p^{M}\right\}$ satisfy a priori estimate 56 .

\subsection{Existence of a solution to the incremental problem}

In this section we show existence of a weak solution:

Theorem 1. Assume the Hypothesis 1 . Then there exists at least one variational solution $\{\mathbf{u}, \varphi, p\} \in$ $V_{U} \times H^{1}(B) \cap K \times V_{P}$ for problem (42)-(50).

Proof. (of Theorem 11) By Proposition 2 there is a solution $\left\{\mathbf{u}^{M}, \varphi^{M}, p^{M}\right\}$ for problem (52)-(54), satisfying a priori estimate (56). Therefore there exists $\{\mathbf{u}, \varphi, p\}$ and a subsequence, denoted by the same superscript, such that

$$
\begin{gathered}
\left\{\mathbf{u}^{M}, \varphi^{M}, p^{M}-p^{b d r y}\right\} \rightarrow\left\{\mathbf{u}, \varphi, p-p^{b d r y}\right\} \quad \text { weakly in } V_{U} \times H^{1}(B) \times V_{P}, \\
\text { strongly in } L^{q}(B)^{5}, q<6, \quad \text { and a.e. on } B, \text { as } M \rightarrow \infty .
\end{gathered}
$$

Obviously $\left(\varphi^{M}-\Phi\right)_{+} \rightarrow 0$, as $M \rightarrow \infty$, and $\varphi \in K$.

Since $\tilde{\varphi}^{M} \rightarrow \tilde{\varphi}$ strongly in $L^{q} B$, for all $q<+\infty$, we pass to the limit in equation 52 without obstacles. Therefore, the triple $\{\mathbf{u}, \varphi, p\}$ satisfies equation $(42)$ and boundary condition (44).

The analogous conclusion holds for variational equation (54) and the triple $\{\mathbf{u}, \varphi, p\}$ satisfies equation (49) and boundary conditions (43), (50).

Next, let $\psi \in K_{+}^{N}=\left\{z \in W^{N}: z(x) \leq 0\right.$ a.e. on $\left.B\right\}, N \leq M$. Then we have

$$
\begin{aligned}
& G_{c} \int_{B}(\left.-\frac{1}{\varepsilon}\left(1-\varphi^{M}\right) \psi+\varepsilon \nabla \varphi^{M} \cdot \nabla \psi\right) d x+\int_{B} M\left(\varphi^{M}-\Phi\right)_{+} \psi d x \\
&+\int_{B} \mathcal{D}\left(\tilde{\varphi}^{M}\right)\left((1-\alpha) p^{M} \operatorname{div} \mathbf{U}+\nabla p^{M} \cdot \mathbf{U}\right) \psi d x+ \\
& \frac{1}{2} \int_{B}\left(\mathcal{A}\left(\tilde{\varphi}^{M}\right) \mathcal{G} e(\mathbf{U}): e(\mathbf{U})-\mathcal{J}\left(\tilde{\varphi}^{M}\right) P\left(2 p^{M}-P\right)\right) \psi d x=0 .
\end{aligned}
$$


Passing to the limit in equation (70) is straightforward and we conclude that

$$
\begin{gathered}
G_{c} \int_{B}\left(-\frac{1}{\varepsilon}(1-\varphi) \psi+\varepsilon \nabla \varphi \cdot \nabla \psi\right) d x \\
+\int_{B} \mathcal{D}(\tilde{\varphi})((1-\alpha) p \operatorname{div} \mathbf{U}+\nabla p \cdot \mathbf{U}) \psi d x+ \\
\frac{1}{2} \int_{B}(\mathcal{A}(\tilde{\varphi}) \mathcal{G} e(\mathbf{U}): e(\mathbf{U})-\mathcal{J}(\tilde{\varphi}) P(2 p-P)) \psi d x \geq 0 .
\end{gathered}
$$

After passing to the limit $N \rightarrow \infty$, we conclude that $\left\{\mathbf{u}, \varphi, p-p^{b d r y}\right\} \in V_{U} \times H^{1}(B) \cap K \times V_{P}$ is a solution to the inequality (45) and (46) is satisfied. It remains to prove the Rice condition (47).

To prove it, we observe that with the choice $\psi=\varphi-\Phi$ inequality (71) holds true. Finally, we choose $\varphi^{M}-\Phi$ as a test function in equation 70 . Using the weak lower semi-continuity of the quadratic terms yields

$$
\begin{gathered}
G_{c} \int_{B}\left(-\frac{1}{\varepsilon}(1-\varphi)(\varphi-\Phi)+\varepsilon \nabla \varphi \cdot \nabla(\varphi-\Phi)\right) d x \\
+\int_{B} \mathcal{D}(\tilde{\varphi})((1-\alpha) p \operatorname{div} \mathbf{U}+\nabla p \cdot \mathbf{U})(\varphi-\Phi) d x+ \\
\frac{1}{2} \int_{B}(\mathcal{A}(\tilde{\varphi}) \mathcal{G} e(\mathbf{U}): e(\mathbf{U})-\mathcal{J}(\tilde{\varphi}) P(2 p-P))(\varphi-\Phi) d x \leq 0 .
\end{gathered}
$$

After comparing (71) and (72), we obtain Rice's condition (47).

\subsection{The Lyapunov functional for the incremental problem}

In this subsection we construct the Lyapunov functional corresponding to the sequence of incremental problems 42 - 50 .

We suppose the quasistatic problem (23)-231) is discretized with a uniform time step $\Delta t$. Given solutions at discrete times $t_{j}, j=0, \ldots, N,\left\{\mathbf{u}_{j}, \varphi_{j}, p_{j}\right\}$, with $\Phi=\varphi_{j-1}, \mathbf{U}=\mathbf{u}_{j-1}$ and $P=p_{j-1}$, are extended from the discrete times $\left\{t_{j}\right\}_{0 \leq j \leq N}$ to $(0, T)$ by

$$
\begin{array}{ccc}
\varphi_{\Delta t}(t)=\varphi_{j} & \text { if } t_{j} \leq t<t_{j+1}, \quad j=0, \ldots N-1 ; \\
& \varphi_{\Delta t}\left(t_{0}\right)=\varphi^{0} ; \\
\mathbf{u}_{\Delta t}(t)=\mathbf{u}_{j} & \text { if } t_{j} \leq t<t_{j+1}, \quad j=0, \ldots N-1 . \\
p_{\Delta t}(t)=p_{j} \quad \text { if } t_{j} \leq t<t_{j+1}, & j=0, \ldots N-1 ; \\
& p_{\Delta t}\left(t_{0}\right)=p^{0} .
\end{array}
$$

$\mathbf{u}_{\Delta t}\left(t_{0}\right) \in V_{U}$ is the solution for 42, (43), 44t). In the formulation we need values at $t-\Delta t$, which correspond to the previous time step. We set

$$
\Phi_{\Delta t}(t)=\varphi_{\Delta t}(t-\Delta t) ; \quad \mathbf{U}_{\Delta t}(t)=\mathbf{u}_{\Delta t}(t-\Delta t) \text { and } P_{\Delta t}(t)=p_{\Delta t}(t-\Delta t) \quad \Delta t \leq t \leq T .
$$

The variational formulation of the incremental problem is then 
Find $\left\{\mathbf{u}_{\Delta t}, \varphi_{\Delta t}, p_{\Delta t}\right\} \in V_{U} \times H^{1}(B) \times V_{P}$ such that $\varphi_{\Delta t}(t) \leq \varphi_{\Delta t}(t-\Delta t)$ for every $t \in(0, T)$ and

$$
\begin{gathered}
\int_{B}\left(A\left(\tilde{\varphi}_{\Delta t}\right) \mathcal{G} e\left(\mathbf{u}_{\Delta t}\right): e(\mathbf{w})+\left(\left(\tilde{\varphi}_{\Delta t}\right)^{2} \nabla p_{\Delta t}+(\alpha-1) \nabla\left(\left(\tilde{\varphi}_{\Delta t}\right)^{2} p_{\Delta t}\right)\right) \cdot \mathbf{w}\right) d x \\
=\int_{\partial_{N} B}\left(\tau_{\Delta t} \cdot \mathbf{w}+p_{\Delta t}^{b d r y} w_{n}\right) d S, \quad \forall \mathbf{w} \in V_{U} \\
G_{c} \int_{B}\left(-\frac{1}{\varepsilon}\left(1-\varphi_{\Delta t}\right) \psi+\varepsilon \nabla \varphi_{\Delta t} \cdot \nabla \psi\right) d x \\
+\int_{B} \mathcal{D}\left(\tilde{\varphi}_{\Delta t}\right)\left(\left((1-\alpha) p_{\Delta t} \operatorname{div} \mathbf{U}_{\Delta t}+\nabla p_{\Delta t} \cdot \mathbf{U}_{\Delta t}\right) \psi d x+\right. \\
\frac{1}{2} \int_{B}\left(\mathcal{A}\left(\tilde{\varphi}_{\Delta t}\right) \mathcal{G} e\left(\mathbf{U}_{\Delta t}\right): e\left(\mathbf{U}_{\Delta t}\right)-\mathcal{J}\left(\tilde{\varphi}_{\Delta t}\right) P_{\Delta t}\left(2 p_{\Delta t}-P_{\Delta t}\right)\right) \psi d x \leq 0 \\
\forall \psi \in H^{1}(B), \psi \geq 0, \text { a.e. on } B \\
G_{c} \int_{B}\left(-\frac{1}{\varepsilon}\left(1-\varphi_{\Delta t}\right)\left(\varphi_{\Delta t}-\Phi_{\Delta t}\right)+\varepsilon \nabla \varphi_{\Delta t} \cdot \nabla\left(\varphi_{\Delta t}-\Phi_{\Delta t}\right)\right) d x \\
+\int_{B} \mathcal{D}\left(\tilde{\varphi}_{\Delta t}\right)\left(\left((1-\alpha) p_{\Delta t} \operatorname{div} \mathbf{U}_{\Delta t}+\nabla p_{\Delta t} \cdot \mathbf{U}_{\Delta t}\right)\left(\varphi_{\Delta t}-\Phi_{\Delta t}\right) d x+\right. \\
\frac{1}{2} \int_{B}\left(\mathcal{A}\left(\tilde{\varphi}_{\Delta t}\right) \mathcal{G} e\left(\mathbf{U}_{\Delta t}\right): e\left(\mathbf{U}_{\Delta t}\right)-\mathcal{J}\left(\tilde{\varphi}_{\Delta t}\right) P_{\Delta t}\left(2 p_{\Delta t}-P_{\Delta t}\right)\right)\left(\varphi_{\Delta t}-\Phi_{\Delta t}\right) d x=0
\end{gathered}
$$

$$
\begin{gathered}
\int_{B} \partial_{\Delta t}\left(c\left(\tilde{\varphi}_{\Delta t}\right) p_{\Delta t}+\operatorname{div}\left(\left(\tilde{\varphi}_{\Delta t}\right)^{2} \mathbf{u}_{\Delta t}\right)+(\alpha-1)\left(\tilde{\varphi}_{\Delta t}\right)^{2} \operatorname{div} \mathbf{u}_{\Delta t}\right) \pi d x \\
+\int_{B} K_{e f f}\left(\tilde{\Phi}_{\Delta t}\right)\left(\nabla p_{\Delta t}-\tilde{\mathbf{G}}\left(\Phi_{\Delta t}\right)\right) \cdot \nabla \pi d x= \\
\int_{B} q_{\Delta t} \pi d x-\int_{\partial_{D} B} v_{\Delta t}^{i n j} \pi d S, \quad \forall \pi \in V_{P}
\end{gathered}
$$

with

$$
\begin{gathered}
\mathcal{A}\left(\tilde{\varphi}_{\Delta t}\right)=\frac{A\left(\tilde{\varphi}_{\Delta t}\right)-A\left(\tilde{\Phi}_{\Delta t}\right)}{\varphi_{\Delta t}-\Phi_{\Delta t}} ; \quad \mathcal{J}\left(\tilde{\varphi}_{\Delta t}\right)=\frac{c\left(\tilde{\varphi}_{\Delta t}\right)-c\left(\tilde{\Phi}_{\Delta t}\right)}{\varphi_{\Delta t}-\Phi_{\Delta t}} \\
\text { and } \mathcal{D}\left(\tilde{\varphi}_{\Delta t}\right)=\frac{\tilde{\varphi}_{\Delta t}^{2}-\tilde{\Phi}_{\Delta t}^{2}}{\varphi_{\Delta t}-\Phi_{\Delta t}}
\end{gathered}
$$

We start with several auxiliary results, which will allow us to construct the Lyapunov functional:

Lemma 1. Let $N \in \mathbb{N}$. Then we have

$$
\begin{gathered}
\Delta t \sum_{n=0}^{N-1} A\left(\tilde{\varphi}_{n+1}\right) \mathcal{G} e\left(\mathbf{u}_{n+1}\right): e\left(\partial_{\Delta t} \mathbf{u}_{n+1}\right)=\frac{1}{2}\left(A\left(\tilde{\varphi}_{N}\right) \mathcal{G} e\left(\mathbf{u}_{N}\right): e\left(\mathbf{u}_{N}\right)-\right. \\
\left.A\left(\tilde{\varphi}_{0}\right) \mathcal{G} e\left(\mathbf{u}_{0}\right): e\left(\mathbf{u}_{0}\right)\right)+\frac{1}{2} \sum_{n=0}^{N-1} A\left(\tilde{\varphi}_{n+1}\right) \mathcal{G} e\left(\mathbf{u}_{n+1}-\mathbf{u}_{n}\right): e\left(\mathbf{u}_{n+1}-\mathbf{u}_{n}\right)- \\
\frac{\Delta t}{2} \sum_{n=0}^{N-1} \mathcal{A}\left(\tilde{\varphi}_{n+1}\right) \mathcal{G} e\left(\mathbf{u}_{n}\right): e\left(\mathbf{u}_{n}\right) \partial_{\Delta t} \varphi_{n+1}
\end{gathered}
$$


Lemma 2. Let $N \in \mathbb{N}$. Then we have

$$
\begin{gathered}
\sum_{n=0}^{N-1}\left(c\left(\tilde{\varphi}_{n+1}\right) p_{n+1}-c\left(\tilde{\varphi}_{n}\right) p_{n}\right) p_{n+1}=\frac{1}{2}\left(c\left(\tilde{\varphi}_{N}\right) p_{N}^{2}-c\left(\tilde{\varphi}_{0}\right)\left(p^{0}\right)^{2}\right)+ \\
\frac{1}{2} \sum_{n=0}^{N-1} c\left(\tilde{\varphi}_{n+1}\right)\left(p_{n+1}-p_{n}\right)^{2}+\frac{1}{2} \sum_{n=0}^{N-1} \mathcal{J}\left(\tilde{\varphi}_{n+1}\right)\left(2 p_{n+1}-p_{n}\right) p_{n} .
\end{gathered}
$$

Lemma 3. Let $N \in \mathbb{N}$. Then for the combination of the cross-terms, which are not multiplied by $(\alpha-1)$, we have

$$
\begin{gathered}
\sum_{n=0}^{N-1}\left(\left(\tilde{\varphi}_{n+1}\right)^{2} \nabla p_{n+1} \partial_{\Delta t} \mathbf{u}_{n+1}+\operatorname{div} \partial_{\Delta t}\left(\left(\tilde{\varphi}_{n+1}\right)^{2} \mathbf{u}_{n+1}\right) p_{n+1}\right. \\
\left.+\mathcal{D}\left(\tilde{\varphi}_{n+1}\right) \partial_{\Delta t} \varphi_{n+1} \mathbf{u}_{n} \nabla p_{n+1}\right)=\sum_{n=0}^{N-1} \operatorname{div}\left(p_{n+1} \partial_{\Delta t}\left(\left(\tilde{\varphi}_{n+1}\right)^{2} \mathbf{u}_{n+1}\right)\right) .
\end{gathered}
$$

Lemma 4. Let $N \in \mathbb{N}$. Then for the combination of the cross-terms, which are multiplied by $(\alpha-1)$, we have

$$
\begin{gathered}
\sum_{n=0}^{N-1}\left(\nabla\left(\left(\tilde{\varphi}_{n+1}\right)^{2} p_{n+1}\right) \partial_{\Delta t} \mathbf{u}_{n+1}+p_{n+1} \partial_{\Delta t}\left(\left(\tilde{\varphi}_{n+1}\right)^{2} \operatorname{div} \mathbf{u}_{n+1}\right)-\right. \\
\left.\mathcal{D}\left(\tilde{\varphi}_{n+1}\right) \partial_{\Delta t} \varphi_{n+1} p_{n+1} \operatorname{div} \mathbf{u}_{n}\right)=\sum_{n=0}^{N-1} \operatorname{div}\left(p_{n+1}\left(\left(\tilde{\varphi}_{n+1}\right)^{2} \partial_{\Delta t} \mathbf{u}_{n+1}\right)\right) .
\end{gathered}
$$

In the considerations which follow we will suppose that $p^{b d r y}=0$. Working with the non-homogeneous pressure boundary condition would lead to long and cumbersome expressions but, on the other hand, their presence would not present any problem.

We introduce the incremental Lyapunov functional (or the incremental free energy functional) by

$$
\begin{gathered}
J^{N}=\frac{1}{2} \int_{B}\left(A\left(\tilde{\varphi}_{N}\right) \mathcal{G} e\left(\mathbf{u}_{N}\right): e\left(\mathbf{u}_{N}\right)+G_{c}\left(\varepsilon\left|\nabla \varphi_{N}\right|^{2}+\frac{1}{\varepsilon}\left(1-\varphi_{N}\right)^{2}\right)\right. \\
\left.+c\left(\tilde{\varphi}_{N}\right) p_{N}^{2}\right) d x-\int_{\partial_{N} B} \tau\left(t_{N}\right) \cdot \mathbf{u}_{N} d S .
\end{gathered}
$$

Then we have the following result

Theorem 2. Let us suppose Hypothesis 1 and, in addition, $p^{b d r y}=0$. Then the following estimate holds:

$$
\begin{gathered}
J^{N}+\Delta t \sum_{n=0}^{N-1} \int_{B} K_{e f f}\left(\tilde{\varphi}_{n}\right) \nabla p_{n+1} \cdot \nabla p_{n+1} d x \leq J^{0}+ \\
\Delta t \sum_{n=0}^{N-1} \int_{B} K_{e f f}\left(\tilde{\varphi}_{n}\right) \tilde{\mathbf{G}}\left(\tilde{\varphi}_{n}\right) \cdot \nabla p_{n+1} d x-\Delta t \sum_{n=0}^{N-1} \int_{\partial_{N} B} \partial_{\Delta t} \tau\left(t_{n+1}\right) \cdot \mathbf{u}_{n+1} d S \\
+\Delta t \sum_{n=0}^{N-1} \int_{\partial_{D} B} v^{i n j}\left(t_{n+1}\right) p_{n+1} d S+\Delta t \sum_{n=0}^{N-1} \int_{B} \tilde{q}\left(t_{n+1}\right) p_{n+1} d x,
\end{gathered}
$$

where $J^{0}$ is calculated using the initial values of the unknowns. 
Proof. We consider system (78)-(81) for times $t_{n}, n=1, \ldots, N$. We test equation (78) by $\partial_{\Delta t} \mathbf{u}_{n+1}$, using equality (80) with $\partial_{\Delta t} \varphi_{n+1}$ and testing equation (81) by $p_{n+1}$. Then we sum up the obtained equalities from $n=0$ to $n=N-1$ and, finally, sum up the three equalities obtained this way. Application of equalities (83)-(86) yields the estimate (88).

\section{Discretization and Solution Algorithm}

For numerical treatment of the whole system, we work with Definition 1 namely equations (52)-(54), in which the setting is time-discretized using a backward difference quotient. Then, we describe spatial discretization that is based on a Galerkin finite element scheme. Finally, we propose a combined Newton method that simultaneously solves for the nonlinear (forward) problem and the enforcement of the crack irreversibility.

\subsection{Aspects of temporal discretization}

All time derivatives are approximated with backward difference quotients as described in the theoretical part of the article. That is:

$$
\partial_{t} \varphi \approx \frac{\varphi-\Phi}{\Delta t}
$$

where $\Phi:=\varphi\left(t^{n-1}\right)$ and the time step size is $\Delta t=t^{n}-t^{n-1}$. In a similar way the time derivative of the pressure equation is formulated.

Moreover, we time-lag in terms of a linear extrapolation $\varphi_{e x}$ of the true $\varphi$ in several terms in order to resolve numerical difficulties related to the indefiniteness of the Jacobian matrix. This is of most importance in the displacement equation. In [13, it has been shown that this procedure is efficient and robust.

\subsection{Spatial discretization}

Spatial discretization is performed with conforming finite elements; namely the discrete spaces consists of bilinears using a mesh decomposed into quadrilaterals. This space is denoted as usually by $Q_{1}^{c}$. The discretization parameter is denoted by $h$ and since the method is conforming, we have $V_{U}^{h} \subset V_{U}$ for the displacements, $W^{h} \subset W$ for the phase-field variable, and $V_{P}^{h} \subset V_{P}$ for the pressure. We employ a Galerkin scheme and formulate a semi-linear form $\mathbf{A}\left(U^{h}\right)\left(\Psi^{h}\right)$ with $U^{h}=\left(\mathbf{u}^{h}, \varphi^{h}, p^{h}\right) \in V_{U}^{h} \times W^{h} \times V_{P}^{h}$ such that the discrete nonlinear system then reads:

Definition 2 (of a single semi-linear form for the penalized formulation). In each time step $t^{n}$, solve for a discrete solution $U^{h}=\left(u^{h}, \varphi^{h}, p^{h}\right) \in V_{U} \times W \times V_{P}$ such that

$$
\begin{gathered}
\mathbf{A}\left(U^{h}\right)\left(\Psi^{h}\right)=\int_{B}\left(A\left(\varphi_{e x}^{h}\right) \mathcal{G} e\left(\mathbf{u}^{h}\right): e\left(\mathbf{w}^{\mathbf{h}}\right)+\left(\left(\varphi_{e x}^{h}\right)^{2} \nabla p^{h}+(\alpha-1) \nabla\left(\left(\varphi_{e x}^{h}\right)^{2} p^{h}\right)\right) \cdot \mathbf{w}^{h}\right) d x-\int_{\partial_{N} B}\left(\tau \cdot \mathbf{w}^{h}+p^{b d r y} w_{n}^{h}\right) d S \\
+G_{c} \int_{B}\left(-\frac{1}{\varepsilon}\left(1-\varphi^{h}\right) \psi^{h}+\varepsilon \nabla \varphi^{h} \cdot \nabla \psi^{h}\right) d x+\int_{B} \delta\left(\varphi^{h}-\Phi\right)_{+} \psi^{h} d x \\
+\int_{B} \mathcal{D}\left(\varphi_{e x}^{h}\right)\left(\left((1-\alpha) p^{h} \operatorname{div} \mathbf{U}+\nabla p^{h} \cdot \mathbf{U}\right) \psi^{h}\right) d x+\frac{1}{2} \int_{B}\left(\mathcal{A}\left(\varphi_{e x}^{h}\right) \mathcal{G} e(\mathbf{U}): e(\mathbf{U})-\mathcal{J}\left(\varphi_{e x}^{h}\right) P\left(2 p^{h}-P\right)\right) \psi^{h} \\
+\int_{B}\left(c\left(\varphi_{e x}^{h}\right) p^{h}+\operatorname{div}\left(\left(\varphi_{e x}^{h}\right)^{2} \mathbf{u}^{h}\right)+(\alpha-1)\left(\varphi_{e x}^{h}\right)^{2} \operatorname{div} \mathbf{u}^{h}\right) \pi^{h} d x \\
+\Delta t \int_{B}\left(K_{e f f}\left(\Phi_{+}\right)\left(\nabla p^{h}-\tilde{\mathbf{G}}\left(\Phi_{+}\right)\right)\right) \cdot \nabla \pi^{h} d x=\Delta t \int_{B} \tilde{q} \pi^{h} d x \\
-\Delta t \int_{\partial_{D} B} v^{i n j} \pi_{r} d S+\int_{B}\left(c\left(\Phi_{+}\right) P+\operatorname{div}\left(\Phi_{+}^{2} \mathbf{U}\right)+(\alpha-1) \Phi_{+}^{2} \operatorname{div} \mathbf{U}\right) \pi_{r} d x
\end{gathered}
$$

for all $\Psi^{h}=\left(\mathbf{w}^{h}, \psi^{h}, \pi^{h}\right) \in V_{U}^{h} \times W^{h} \times V_{P}^{h}$. 
Remark 4 (Treatment of the crack irreversibility condition). The crack irreversibility can be either treated via penalization using the parameter $\delta$ as it has been used for related problems of pressurized and fluid-filled fractures [17, 22]; or as recently proposed in [13], employing a robust primal-dual active set strategy that can be interpreted as a semi-smooth Newton method [14]. Further aspects are explained in more detail below in Section 6.4.

\subsection{Newton's method for the nonlinear forward problem}

In this section, we restrict our attention to the nonlinear solution of the main equations (i.e., the forward problem). We apply Newton's method to the fully-coupled problem in order to identify optimal convergence rates. Here, the Jacobian matrix is derived analytically in order to identify optimal Newton convergence whenever possible. The semi-linear form of the forward problem is defined in Definition 2 and neglects the penalization term. The nonlinear iteration reads:

Algorithm 1 (Newton iteration of the fully-coupled system). At a given time level; repeat the Newton iterations for $k=0,1,2, \ldots$ :

1. Find $d U_{k}^{h}:=\left\{d \mathbf{u}_{k}^{h}, d \varphi_{k}^{h}, d p_{k}^{h}\right\}$ by solving the linear system

$$
\mathbf{A}^{\prime}\left(U_{k}^{h}\right)\left(d U_{k}^{h}, \Psi^{h}\right)=-\mathbf{A}\left(U_{k}^{h}\right)\left(\Psi^{h}\right) \quad \forall \Psi \in V^{h} \times W^{h} \times V^{h} .
$$

2. Find a step size $0<\omega \leq 1$ using line search (see Remark 5) to get

$$
U_{k+1}^{h}=U_{k}^{h}+\omega d U_{k}^{h}
$$

with $\mathbf{A}\left(U_{k+1}^{h}\right)\left(\Psi^{h}\right)<\mathbf{A}\left(U_{k}^{h}\right)\left(\Psi^{h}\right)$.

Finish the Newton loop if the stopping criteria is fulfilled:

$$
\left|\mathbf{A}\left(U_{k}^{h}\right)\left(\Psi^{h}\right)\right|<\text { TOL . }
$$

In Algorithm 1, the Jacobian is derived by computing the Gâteaux derivative. The application to a semi-linear form reads:

$$
\mathbf{A}^{\prime}(U)(d U, \Psi):=\lim _{s \rightarrow 0} \frac{1}{s}\{\mathbf{A}(U+s d U)(\Psi)-\mathbf{A}(U)(\Psi)\}=\left.\frac{d}{d s} \mathbf{A}(U+s d U)(\Psi)\right|_{s=0} .
$$

The Jacobian to Definition 2 reads:

$$
\begin{gathered}
\mathbf{A}^{\prime}\left(U^{h}\right)\left(d U^{h}, \Psi^{h}\right)=\int_{B}\left(A\left(\varphi_{e x}^{h}\right) \mathcal{G} e\left(\mathbf{d} \mathbf{u}^{h}\right): e\left(\mathbf{w}^{\mathbf{h}}\right)+\left(\left(\varphi_{e x}^{h}\right)^{2} \nabla d p^{h}+(\alpha-1) \nabla\left(\left(\varphi_{e x}^{h}\right)^{2} d p^{h}\right)\right) \cdot \mathbf{w}^{h}\right) d x \\
\quad+G_{c} \int_{B}\left(\frac{1}{\varepsilon}\left(d \varphi^{h}\right) \psi^{h}+\varepsilon \nabla d \varphi^{h} \cdot \nabla \psi^{h}\right) d x \\
+\int_{B} \mathcal{D}\left(\varphi_{e x}^{h}\right)\left(\left((1-\alpha) d p^{h} \operatorname{div} \mathbf{U}+\nabla d p^{h} \cdot \mathbf{U}\right) \psi^{h}\right) d x+ \\
\quad \frac{1}{2} \int_{B}\left(-\mathcal{J}\left(\varphi_{e x}^{h}\right) P\left(2 d p^{h}\right)\right) \psi^{h} \\
+\int_{B}\left(c\left(\varphi_{e x}^{h}\right) d p^{h}+\operatorname{div}\left(\left(\varphi_{e x}^{h}\right)^{2} \mathbf{d} \mathbf{u}^{h}\right)+(\alpha-1)\left(\varphi_{e x}^{h}\right)^{2} \operatorname{div} \mathbf{d} \mathbf{u}^{h}\right) \pi^{h} d x \\
+\Delta t \int_{B}\left(K_{e f f}\left(\Phi_{+}\right)\left(\nabla d p^{h}-\tilde{\mathbf{G}}\left(\Phi_{+}\right)\right)\right) \cdot \nabla \pi^{h} d x=0
\end{gathered}
$$

for all $\Psi^{h}=\left(\mathbf{w}^{h}, \psi^{h}, \pi^{h}\right) \in V_{U}^{h} \times W^{h} \times V_{P}^{h}$. 
Remark 5 (Backtracking line search). A crucial role for (highly) nonlinear problems includes the appropriate determination of $\omega$. A simple backtracking strategy is employed in the present paper and consists of modifying the update step in (91) as follows: For given $\omega \in(0,1)$ (in our numerical tests, we choose $\omega=0.6$ ) determine the minimal $l^{*} \in \mathbb{N}$ via $l=0,1, \ldots, N_{l}$, such that

$$
\begin{aligned}
R\left(U_{k+1, l}^{h}\right) & <R\left(U_{k, l}^{h}\right), \\
U_{k+1, l}^{h} & =U_{k+1}^{h}+\omega^{l} d U_{k}^{h} .
\end{aligned}
$$

For the minimal $l^{*}$, we set

$$
U_{k+1}^{h}:=U_{k+1, l^{*}}^{h}
$$

In this context, the nonlinear residual $R(\cdot)$ is defined as

$$
R\left(U^{h}\right):=\max _{i}\left\{\mathbf{A}\left(U^{h}\right)\left(\Psi_{i}\right)-\hat{F}\left(\Psi_{i}\right)\right\} \quad \forall U^{h}=\left(\mathbf{w}^{h}, \psi^{h}, \pi^{h}\right) \in V_{U}^{h} \times W^{h} \times V_{P}^{h},
$$

where $\left\{\Psi_{i}\right\}$ denotes the nodal basis of $V_{U}^{h} \times W^{h} \times V_{P}^{h}$. This algorithm works quite well for our problems and is applied to both the nonlinear forward model and the semi-smooth Newton method to realize the primal-dual active set.

\subsection{A combined Newton method for the variational inequality system}

We combine the Newton solver from the previous section with a semi-smooth Newton iteration for treating the crack irreversibility. Such an algorithm has been proposed in [13. Instead of penalizing the equations to solve the variational inequality, key idea of the primal-dual active set algorithm is that the domain is partitioned into an active set and an inactive set. In the active set, the constraint (here $\varphi=\Phi)$ is active, whereas in the inactive set we have $\varphi<\Phi$ and we solve the PDE. The interpretation as a semi-smooth Newton method allows detailed convergence analysis [14] and fast convergence; i.e. a locally superlinear convergence.

With these preparations, we extend Algorithm 1 and define the combined Newton active-set iteration:

Algorithm 2 (Combined Newton of the fully-coupled system). At a given time level; repeat the Newton iterations for $k=0,1,2, \ldots$ :

1. Assemble the residual $\mathbf{A}\left(U_{k}^{h}\right)\left(\Psi^{h}\right)$ with $U_{k}^{h}=\left(\mathbf{u}_{k}^{h}, \varphi_{k}^{h}, p_{k}^{h}\right)$ formulated in Definition 2 consisting of displacements, phase-field, and pressure

2. Compute active set $\mathcal{A}_{k}$ (see Remark[6] and [13]).

3. Assemble matrix $\mathbf{A}^{\prime}\left(U_{k}^{h}\right)\left(\cdot, \Psi^{h}\right)$ and right-hand side $-\mathbf{A}\left(U_{k}^{h}\right)\left(\Psi^{h}\right)$

4. Eliminate rows and columns in active set $\mathcal{A}_{k}$ from matrix and right hand side to obtain $\widetilde{\mathbf{A}}^{\prime}\left(U_{k}^{h}\right)\left(\cdot, \Psi^{h}\right)$ and $-\widetilde{\mathbf{A}}\left(U_{k}^{h}\right)\left(\Psi^{h}\right)$

5. Solve the linear system using a GMRES method with block-diagonal preconditioning; namely find $d U_{k}^{h}$ such that

$$
\widetilde{\mathbf{A}}^{\prime}\left(U_{k}^{h}\right)\left(d U_{k}^{h}, \Psi^{h}\right)=-\widetilde{\mathbf{A}}\left(U_{k}^{h}\right)\left(\Psi^{h}\right) \quad \forall \Psi \in V^{h} \times W^{h} \times V^{h} .
$$


6. Find a step size $0<\omega \leq 1$ using the line search algorithm explained in Remark 5 to compute

$$
U_{k+1}^{h}=U_{k}^{h}+\omega d U_{k}^{h}
$$

with $\widetilde{A}\left(U_{k+1}^{h}\right)\left(\Psi^{h}\right)<\widetilde{A}\left(U_{k}^{h}\right)\left(\Psi^{h}\right)$.

Finish the Newton loop if both stopping criteria are fulfilled simultaneously:

$$
\mathcal{A}_{k+1}=\mathcal{A}_{k} \quad \text { and } \quad \widetilde{\mathbf{A}}\left(U_{k}^{h}\right)\left(\Psi^{h}\right)<\text { TOL } .
$$

Remark 6 (Computing the active set). Determining the active set follows the procedure as it has been described, for example, in 14]. On the discrete level, we check at each degree of freedom if the index $i$ is in the active set $\mathcal{A}_{k}$ :

$$
\left(B^{-1}\right)_{i i}\left(R\left(U_{k}^{h}\right)\right)_{i}+c\left(d U_{h}^{k}\right)_{i}>0 .
$$

Otherwise, the index belongs to the inactive set $\mathcal{I}$. Here, $B$ is a mass matrix (details in $[14]), R\left(U_{k}^{h}\right)$ the nonlinear residual from before, $c>0$ a constant, and $d U_{h}^{k}$ Newton's update. Each row and corresponding column of degree of freedoms $i$ that are part of the active set are then eliminated from the Jacobian $\mathbf{A}^{\prime}\left(U_{k}^{h}\right)\left(\cdot, \Psi^{h}\right)$ and the right hand side $\mathbf{A}\left(U_{k}^{h}\right)\left(\Psi^{h}\right)$ and thus the reduced systems are denoted by $\widetilde{\mathbf{A}}^{\prime}\left(U_{k}^{h}\right)\left(d U_{k}^{h}, \Psi^{h}\right)$ and $\widetilde{\mathbf{A}}\left(U_{k}^{h}\right)\left(\Psi^{h}\right)$, respectively.

Inside Algorithm 2, we solve the linear equations with the generalized minimal residual (GMRES) method and block-diagonal preconditioning as implemented in [13] for the fully-coupled solution for $\{\mathbf{u}, \varphi\}$. That program has been extended to solve the $\{\mathbf{u}, \varphi, p\}$ system. The (reduced) linear equation system 93$)(A d U=b)$ has the following structure:

$$
\left(\begin{array}{ccc}
A_{u u} & 0 & B_{u p} \\
C_{\varphi u} & A_{\varphi \varphi} & M_{\varphi p} \\
B_{p u} & 0 & A_{p p}
\end{array}\right)\left(\begin{array}{l}
\mathbf{d} \mathbf{u}_{k}^{h} \\
d \varphi_{k}^{h} \\
d p_{k}^{h}
\end{array}\right)=\left(\begin{array}{c}
\mathbf{b}_{u} \\
b_{\varphi} \\
b_{p}
\end{array}\right) .
$$

Here, the right hand side values $\left\{\mathbf{b}_{u}, b_{\varphi}, b_{p}\right\}$ are assembled in terms of Newton's (reduced) residual $\widetilde{\mathbf{A}}\left(U_{k}^{h}\right)\left(\Psi^{h}\right)$. The terms in the Jacobian matrix are specified through assembling the directional derivatives in 6.3). Let $N_{u}, N_{\varphi}$ and $N_{p}$ be the dimensions of the spaces $V_{u}^{h}, W^{h}$ and $V_{p}^{h}$. Then,

$$
\begin{aligned}
A_{u u}= & \left(A\left(\varphi_{e x}^{h}\right) \mathcal{G} e\left(\mathbf{w}^{\mathbf{j}}\right): e\left(\mathbf{w}^{\mathbf{i}}\right)\right)_{i, j=1}^{N_{u}, N_{u}}, \\
B_{u p}= & \left(\left(\varphi_{e x}^{h}\right)^{2} \nabla \pi^{j}+(\alpha-1) \nabla\left(\left(\varphi_{e x}^{h}\right)^{2} \pi^{j}\right) \cdot \mathbf{w}^{i}\right)_{i, j=1}^{N_{u}, N_{p}}, \\
C_{\varphi u}= & 0, \\
A_{\varphi \varphi}= & G_{c}\left(\left(\frac{1}{\varepsilon}\left(\psi^{j}\right) \psi^{i}+\varepsilon \nabla \psi^{j} \cdot \nabla \psi^{i}\right)\right)_{i, j=1}^{N_{\varphi}, N_{\varphi}}, \\
M_{\varphi p}= & \left(\mathcal{D}\left(\varphi_{e x}^{h}\right)\left(\left((1-\alpha) \pi^{j} \operatorname{div} \mathbf{U}+\nabla \pi^{j} \cdot \mathbf{U}\right) \psi^{i}\right)\right. \\
& \left.-\left(\left(\mathcal{J}\left(\varphi_{e x}^{h}\right) P\left(2 \pi^{j}\right)\right) \psi^{i}\right)\right)_{i, j=1}^{N_{\varphi}, N_{p}}, \\
B_{p u}= & \left(\left(\operatorname{div}\left(\left(\varphi_{e x}^{h}\right)^{2} \mathbf{w}^{j}\right)+(\alpha-1)\left(\varphi_{e x}^{h}\right)^{2} \operatorname{div} \mathbf{w}^{j}\right) \pi^{i}\right)_{i, j=1}^{N_{p}, N_{u}}, \\
A_{p p}= & \left(\left(K_{e f f}\left(\Phi_{+}\right)\left(\nabla \pi^{j}-\tilde{\mathbf{G}}\left(\Phi_{+}\right)\right)\right) \cdot \nabla \pi^{i}\right)_{i, j=1}^{N_{p}, N_{p}} .
\end{aligned}
$$

The structure of the Jacobian $\widetilde{\mathbf{A}}^{\prime}\left(U_{k}^{h}\right)\left(d U_{k}^{h}, \Psi^{h}\right)$ is important since with this information, a preconditioner $P^{-1}$ can be constructed such that $P^{-1} A$ has a moderate condition number. Here we simply choose

$$
P^{-1}=\left(\begin{array}{ccc}
A_{u u}^{-1} & 0 & 0 \\
0 & A_{\varphi \varphi}^{-1} & 0 \\
0 & 0 & A_{p p}^{-1}
\end{array}\right) .
$$


This preconditioner is computed with an algebraic multigrid method from Trilinos [23] and yields a moderate number of iterations of the GMRES solver. For the examples in this paper we observed between 10 and 90 GMRES iterations per Newton step.

\section{Numerical tests}

In this final section, three different configurations of fluid-filled fractures in poroelasticity are considered. Specifically, Biot's coefficient is chosen as $\alpha=1$. All remaining parameters are summarized in Table 2 ,

\begin{tabular}{|l|l|l|l|}
\hline \hline$S Y M B O L$ & QUANTITY & VALUE & UNITS (in SI) \\
\hline$k$ & bulk regularization & $10^{-12}$ & $\mathrm{~m}$ \\
\hline$\varepsilon$ & phase-field regularization & 0.088 & $\mathrm{~m}$ \\
\hline$h$ & discretization parameter & $0.088,0.044,0.022$ & $\mathrm{~m}$ \\
\hline$\Delta t$ & time step size & 0.01 & $\mathrm{~s}$ \\
\hline$T$ & Total number of time steps & $30(\mathrm{ex} \cdot 1), 50(\mathrm{ex} .2,3)$ & \\
\hline$\alpha$ & Biot coefficient & 1 & \\
\hline$K_{R}$ & Reservoir permeability & $10^{-11}(\mathrm{ex} \cdot 1,2), 10^{-14}-10^{-11}(\mathrm{ex} .3)$ & Darcy \\
\hline$K_{F}$ & Fracture permeability & $w^{2} / 12$ in the propagation direction & Darcy \\
\hline$w$ & Fracture width (COD, aperture) & $w=[u \cdot n]$ & $\mathrm{m}$ \\
\hline$c_{R}$ & Reservoir fluid compressibility & $10^{-8}$ & $1 / \mathrm{Pa}$ \\
\hline$c_{F}$ & Fracture fluid compressibility & $10^{-8}$ & $1 / \mathrm{Pa}$ \\
\hline$\eta_{R}$ & Reservoir fluid viscosity & $10^{-3}$ & $\mathrm{~kg} / \mathrm{m} \mathrm{sec}$ \\
\hline$\eta_{F}$ & Fracture fluid viscosity & $10^{-3}$ & $\mathrm{~kg} / \mathrm{m} \mathrm{sec}$ \\
\hline$p_{p b}$ & Injection pressure & $10^{-8}$ & $\mathrm{~Pa}$ \\
\hline$E$ & Young's modulus & $10^{8}$ & $\mathrm{~Pa}$ \\
\hline$\nu_{S}$ & Poisson's ratio & 0.2 & \\
\hline$G_{c}$ & Critical energy release rate & 1 & $\mathrm{~J} / \mathrm{m}^{2}$ \\
\hline
\end{tabular}

Table 2: Parameters for the numerical examples

The domain is $\Omega=(0,4)^{2} m$ with boundary $\partial \Omega$. As boundary conditions, we describe $u=0$ on $\partial \Omega, \partial_{n} \varphi=0$ on $\partial \Omega$ and $\partial_{n} p=0$ on $\partial \Omega$ (no flow). In order to study the robustness of our numerical solutions, we compute the first two examples on three different meshes using global mesh refinement. We emphasize that $\varepsilon=0.088 \mathrm{~m}$ is fixed such that we are able to study $h$ convergence.

A point source injection is modeled as $q=p_{p h} \frac{d}{\pi} \exp \left(-d *\left(x-x_{0}\right)^{2}\right)$ with $d=10000$ with $x_{0}=(2,2)$. The fracture permeability is computed as $K_{F} \sim w^{2} / 12 \eta_{F}$ where $w$ is the width of the fracture; for a detailed derivation, we refer to [17]. The function $c\left(\varphi_{+}\right)$is a linear interpolation with $c(0)=c_{F}$ and $c(1)=\frac{1}{M}$.

As functionals, we observe the crack length and the maximum pressure over time.

\subsection{A straight propagating fracture}

In this first example, an initial crack is described with length $l_{0}=0.4$ on $\mathcal{C}=(1.8-h, 2.2+h) \times(2-$ $h, 2-h) \subset \Omega$. A single-phase fluid with source term $q$ is injected into the middle of the domain in the point $(2,2)$.

Our findings can be observed in Figures 2 - 5. The crack pattern is displayed in Figure 2, The pressure fields of the fracture and the reservoir interact. Specifically, the pressure at the tips of the 
fracture is very low and even becomes negative as observed in Figure 3 . These findings are confirmed in Figure 4 in which the pressure is plotted over the middle cross section. This behavior has been already discussed in the literature by others $[8,19,18$. The displacement field are illustrated in Figure 5. We observe the typical shape of the crack opening displacement; namely zero opening at the tips of the fracture and largest opening in the middle. This phenomenon was predicted by the theoretical calculations made in [20] in their Section 2.4 for 2D configurations. Finally, we observe in Figure 6 the crack length and the total pressure on three different meshes in order to study the robustness of our solutions. Our findings indicate that our method is computationally stable for $h$-refinement while keeping $\varepsilon$ fixed.
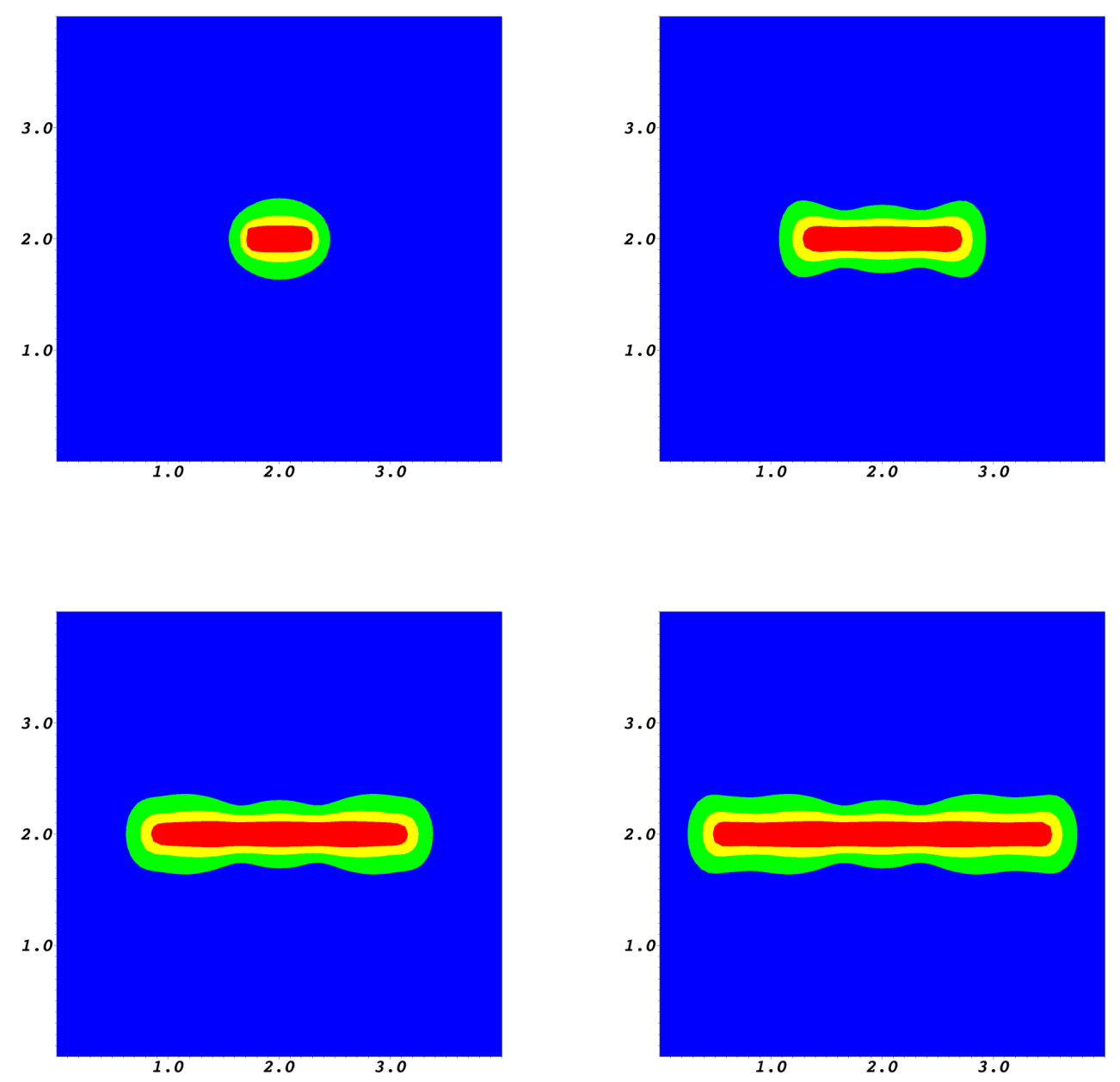

Figure 2: Example 1: Crack pattern at times $T=1,10,20,30 s$ using the phase-field variable $\varphi$. In the crack (red colored) region $\varphi=0$ and in the unbroken material, $\varphi=1$. In between $0<\varphi<1$, which denotes the smooth transition zone. 

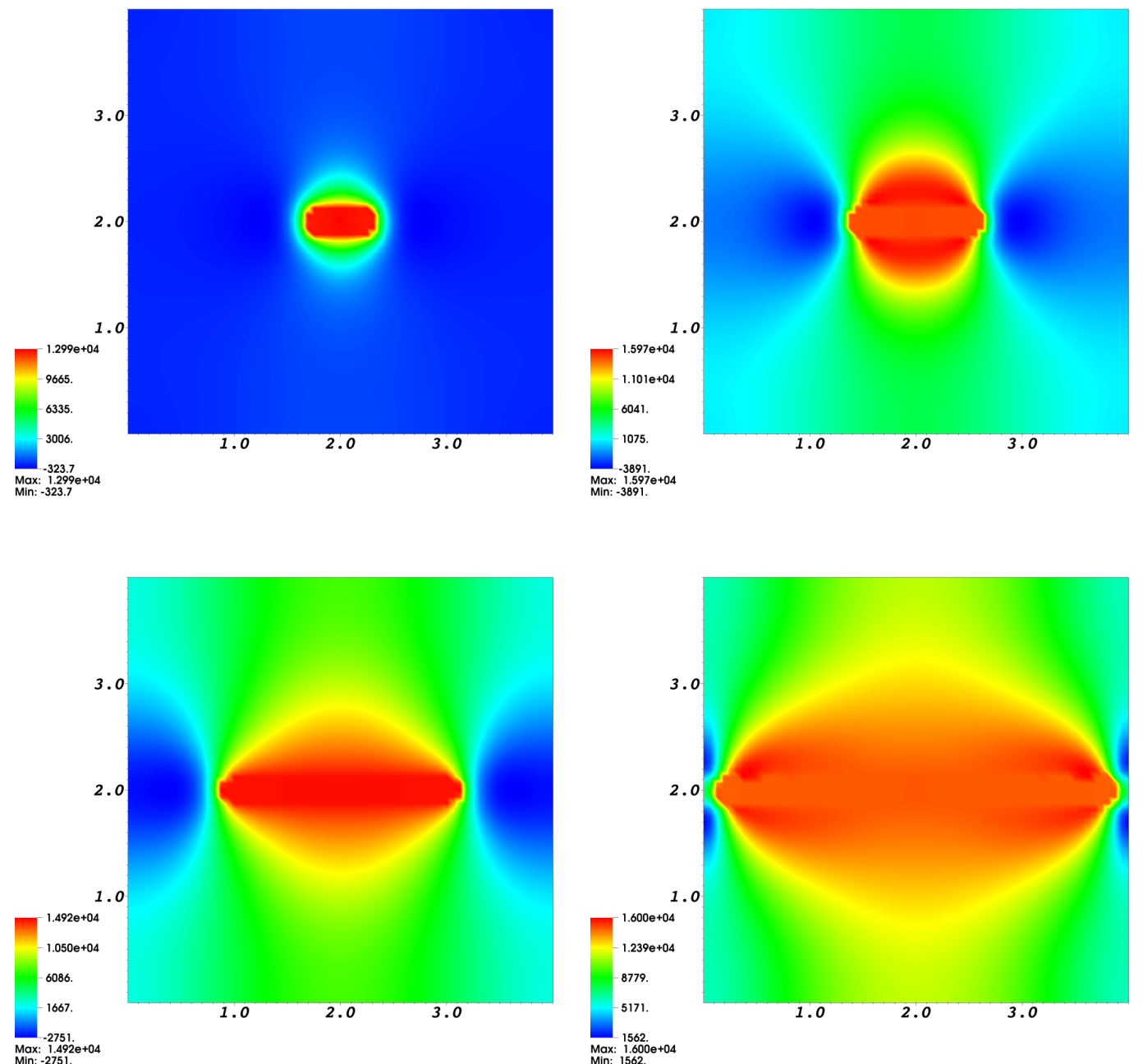

Figure 3: Example 1: Pressure (in $P a$ ) at times $T=1,10,30,40 \mathrm{~s}$. Here, we observe a large pressure difference with negative pressures in front of the tips of the fracture. 

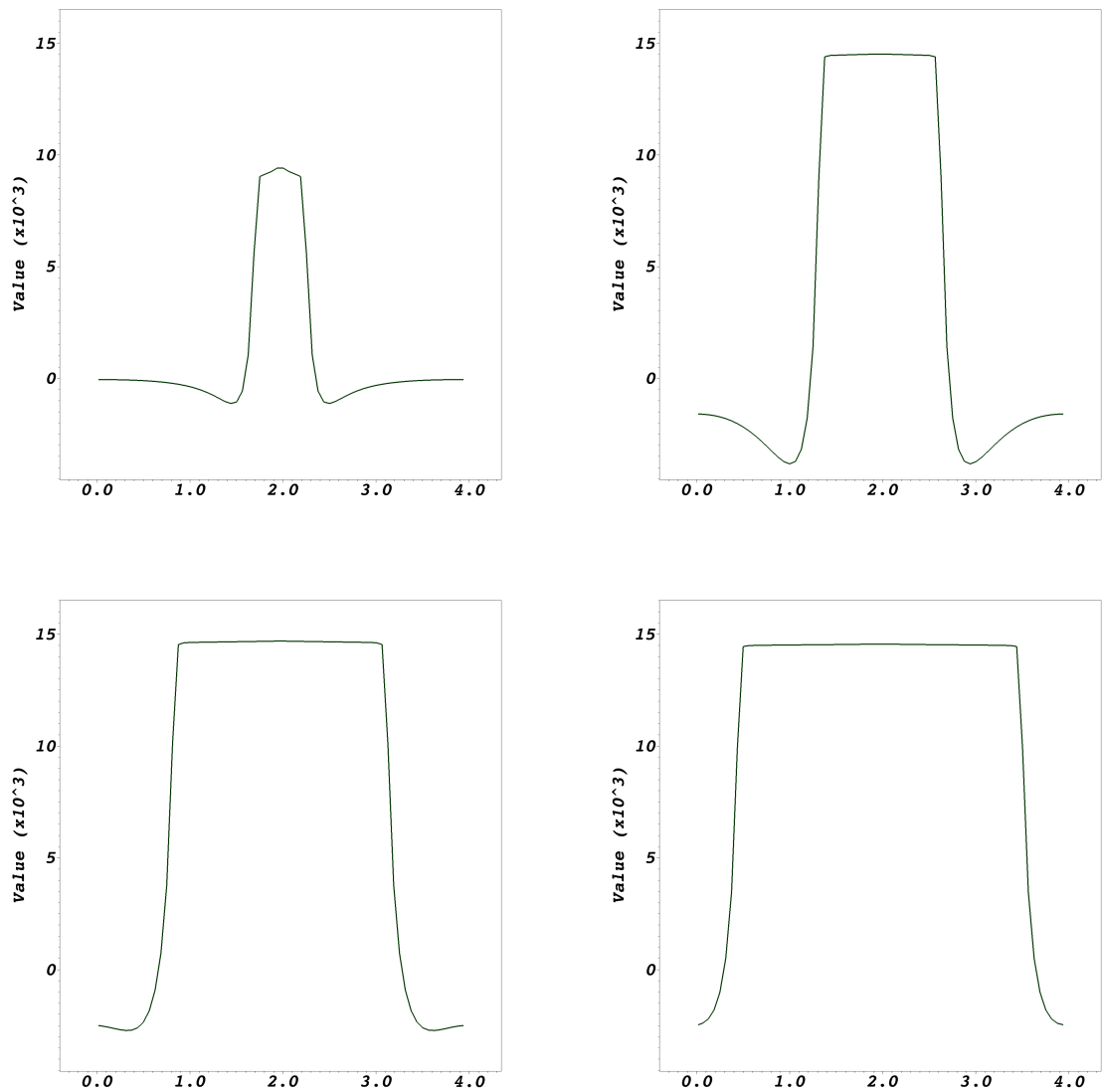

Figure 4: Example 1: Pressure plot over the cross section $\{(x, 2) \mid 0 \leq x \leq 4\}$ at times $T=1,10,30,40 s$. The units of horizontal and vertical axes are $m$ and $P a$, respectively. Here, we observe large pressures in the fracture and low (negative) pressures at the (propagating) tips. As long as the fracture is not propagating, i.e., between $T=1 s$ and $T=10 \mathrm{~s}$, the pressure increases. While the fracture is propagating we observe a slight pressure decrease. In addition, while the fracture is propagating negative pressures at the tips remain but they do not decrease further.
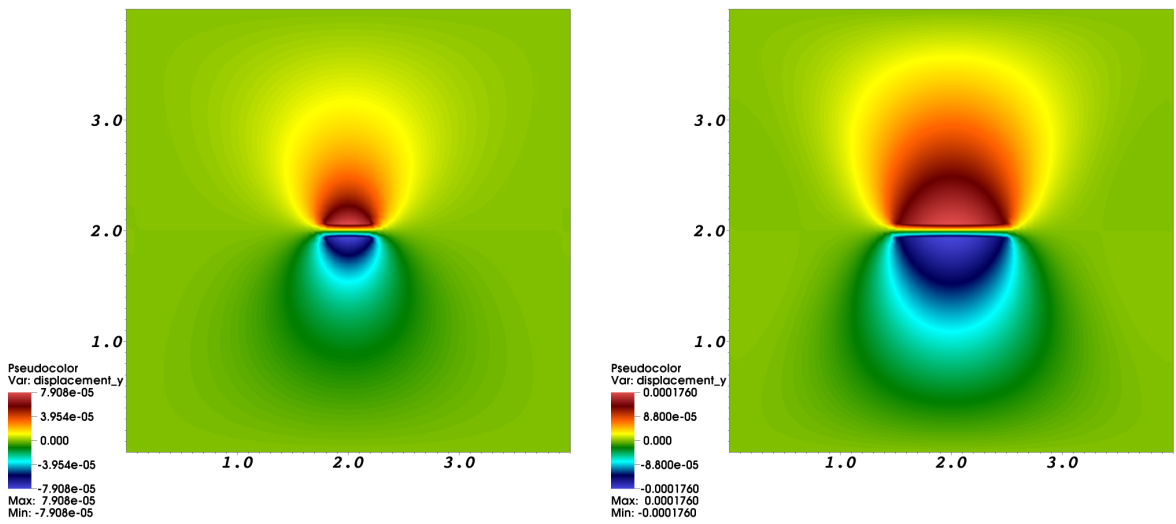

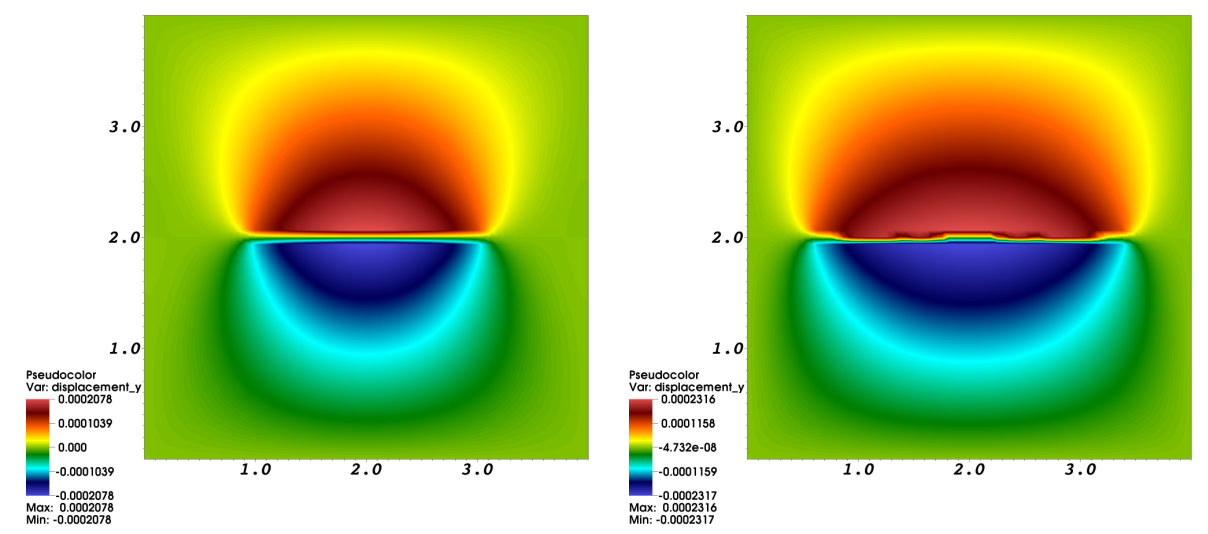

Figure 5: Example 1: $y$-displacement field (in $m$ ) at times $T=1,10,30,40 s$. Here, the displacements are zero at the tips of the fracture and the highest value is obtained in the middle. As in the first example, this corresponds to the theoretical calculations of [20] in their Section 2.4.
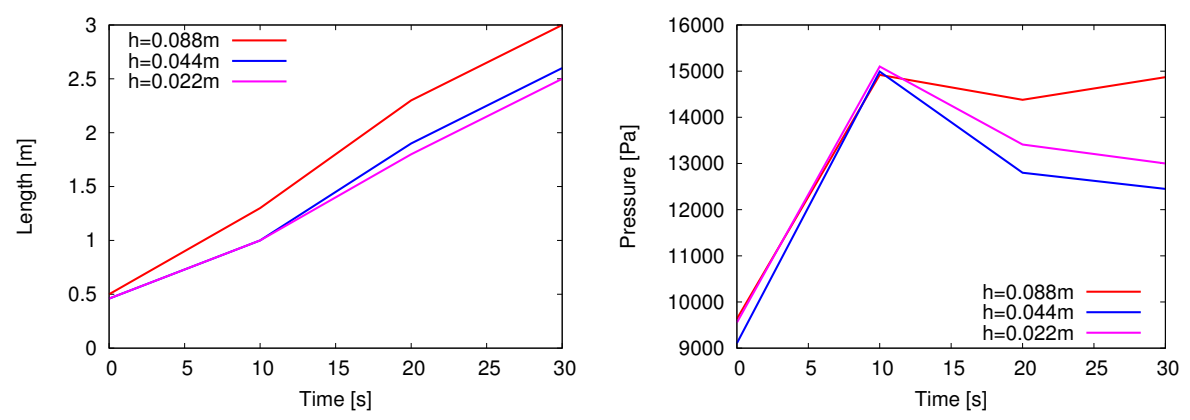

Figure 6: Example 1: Convergence studies of crack length and maximum pressure versus time on three different mesh levels. For fixed $\varepsilon$, we observe $h$-convergence in the length as well as in the pressure. In particular, we observe a pressure drop while the crack is growing. On the coarse mesh the pressure again increases while the pressure is monotonically decreasing on the two fine meshes.

\subsection{Propagation of multiple fractures in a homogeneous porous medium}

In this second test, the domain, boundary conditions and the first fracture (the horizontal one) remain the same as in the first example. We now add a second, vertically-aligned fracture on $(2.6 \pm h, 2.0 \pm$ $0.2) \mathrm{m}$. We only do inject a fluid into fracture 1 (like in the first example). Consequently, the second fracture can be seen as a natural non-pressurized fracture.

Analyzing our findings, the following conclusions can be inferred: In Figure 7, we observe the crack pattern evolution and see that only fracture 1 starts propagating because fracture 2 is not pressurized. They join at time $T=7$. Next, the horizontal crack continues further propagating. Later, the second crack also starts propagating. That fracture 2 is not pressurized can be justified in Figure 8 since high pressure is in the first time steps only being seen in fracture 1 . When they join, also fracture 2 is pressurized and fluid flows inside. Similarly to Example 7.1, the pressure field is negative at the tips of the fractures. Finally, convergence studies of the half crack length of the horizontal fracture and maximum pressure versus time on three different mesh levels are shown in Figure 9. 

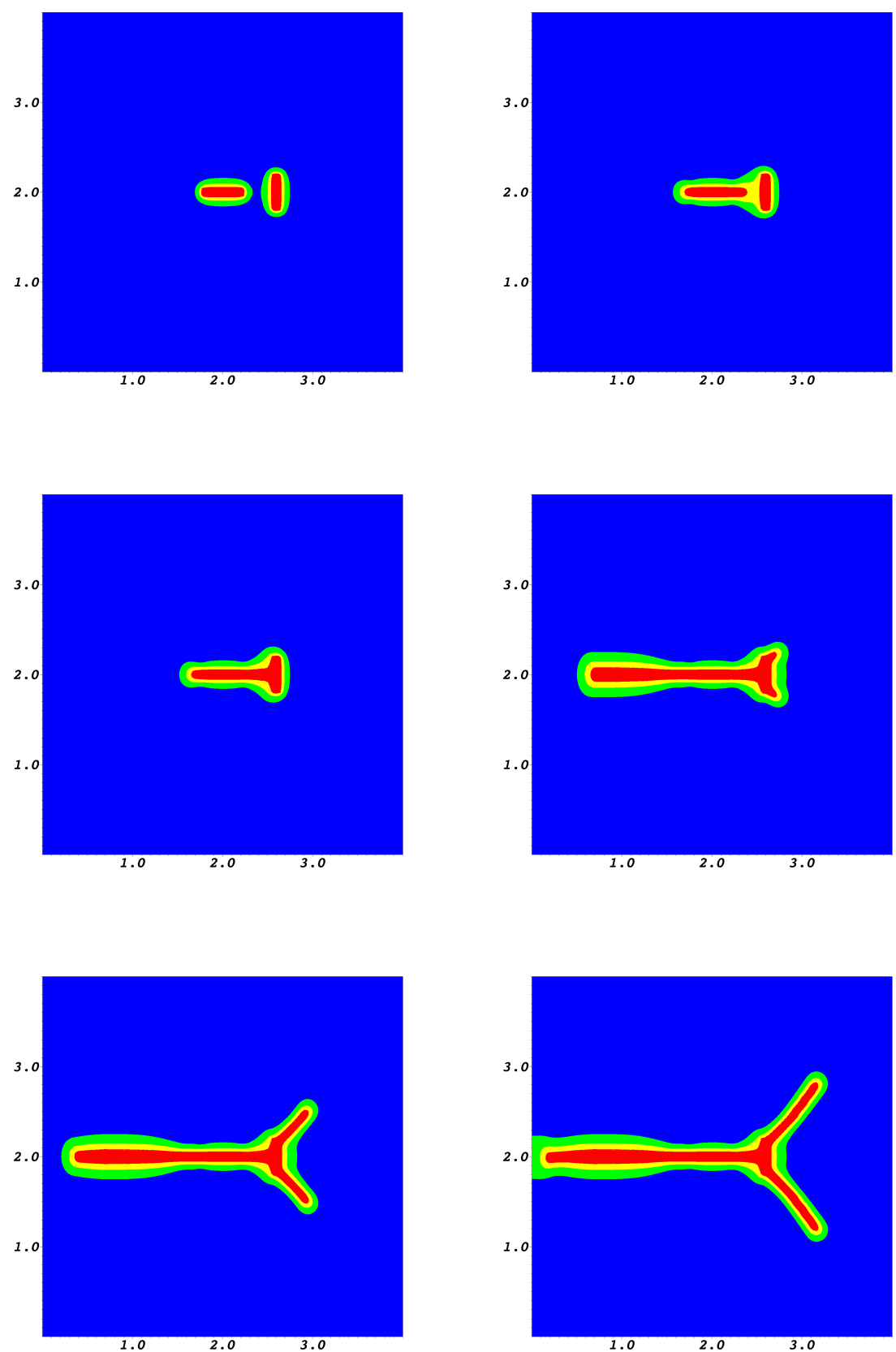

Figure 7: Example 2: Crack pattern at times $T=1,6,7,20,30,50 \mathrm{~s}$ with two fractures. Fracture 1 (the horizontal fracture) joins fracture 2 (the vertical fracture) at $T=7 \mathrm{~s}$. The crack pattern is displayed with the help of the phase-field variable $\varphi$. In the crack (red colored) region $\varphi=0$ and in the unbroken material, $\varphi=1$. In between $0<\varphi<1$, which denotes the smooth transition zone. 

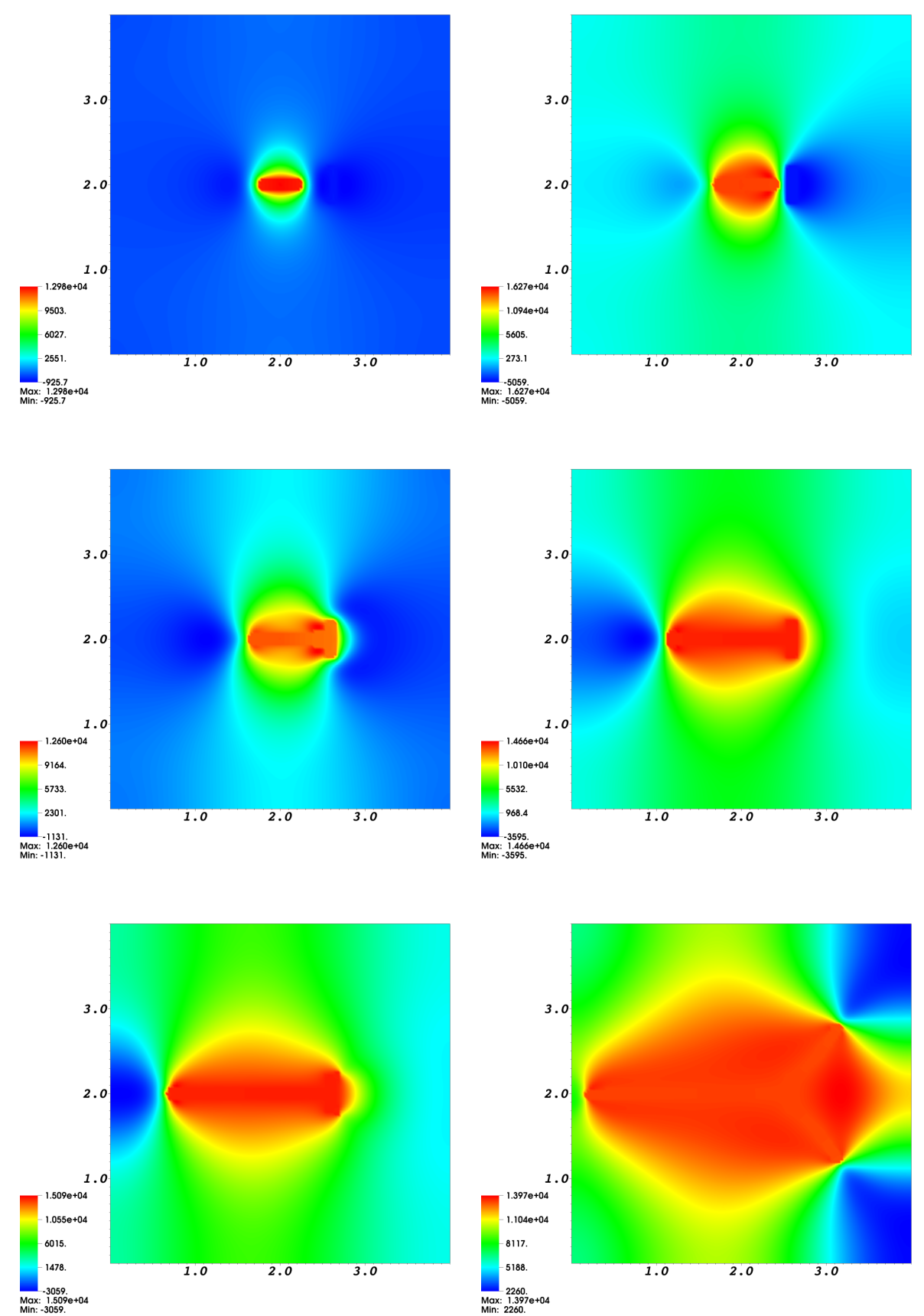

Figure 8: Example 2: Pressure (in $P a$ ) at times $T=1,6,7,20,30,50 \mathrm{~s}$. Again, we observe a large pressure difference with low pressures (in blue color) in front of the fracture tips. In fact, the pressure is lowest first at right when fracture 1 joins fracture 2 . Then, fracture 1 further grows and we observe lowest pressure at $T=20 \mathrm{~s}$ at left in front of the growing fracture. Later, the two fracture tips of the second fracture start growing and now the lowest pressures are seen here at $T=50 \mathrm{~s}$. In this final figure properly the pressure might be still very low for the fracture 1 tip at left, but this fracture is leaving the domain in this moment. Here, we notice that boundary interactions/influences are still subject of current research. But in the last figure, our primary focus is on the tips of fracture 2 . 

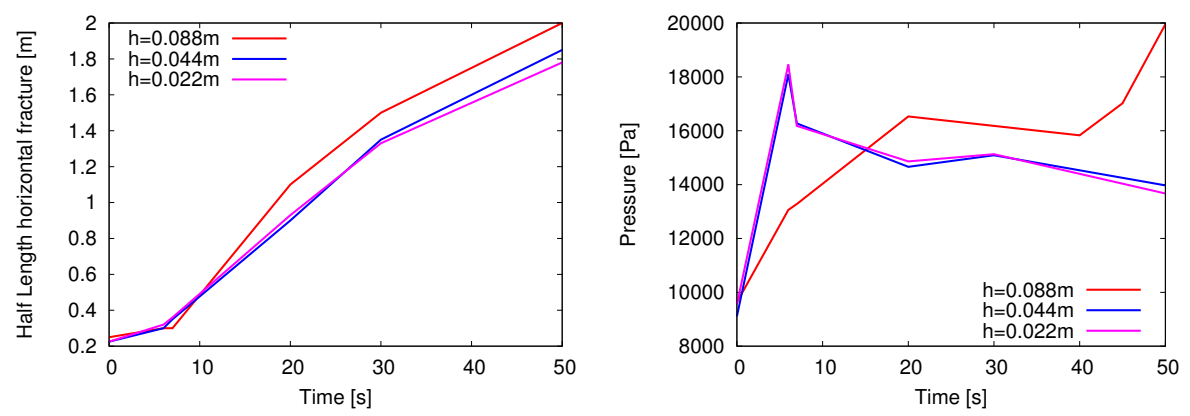

Figure 9: Example 2: Convergence studies of half crack length of the horizontal fracture and maximum pressure versus time on three different mesh levels. For fixed $\varepsilon$, we observe $h$-convergence in the length as well as in the pressure. In particular, we observe a huge pressure drop at $T=7 s$ when the horizontal crack joins the vertical crack. In this example, we also observe that a too coarse mesh does might give wrong answers as here seen for the pressure. The pressure increase towards $T=50$ is due to the fact that this crack reaches the boundary and lead to disputable results.

\subsection{Fracture propagation in a heterogeneous porous medium}

In this final example, the reservoir permeability $K_{R}$ varies. This is an important example motivated by practical applications. Examples with varying geomechanics parameters have been presented in [16, 13] as well as in [24] to couple phase-field fracture propagation with a reservoir simulator.

Except of the reservoir permeability, the configuration, boundary conditions, model and material parameters are the same as in Example 7.1. Analyzing our results, we observe that the fracture now grows on a non-planar way due to the non-constant permeability field (see Figure 11). The pressure field is displayed in Figure 12. Again, low pressures occur at the tips of the fracture.
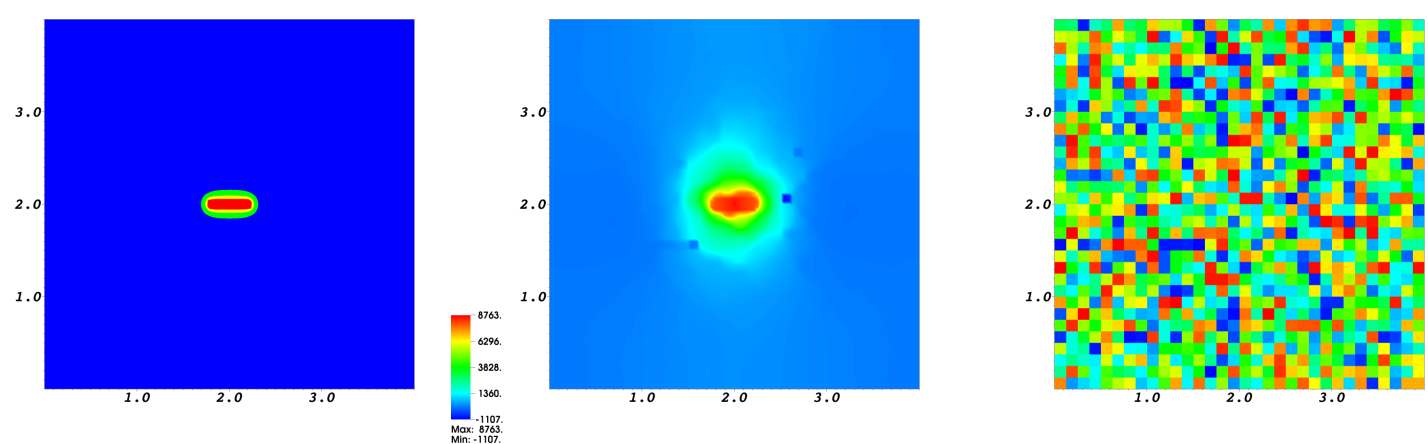

Figure 10: Example 3: Initial crack, pressure and permeability distribution 

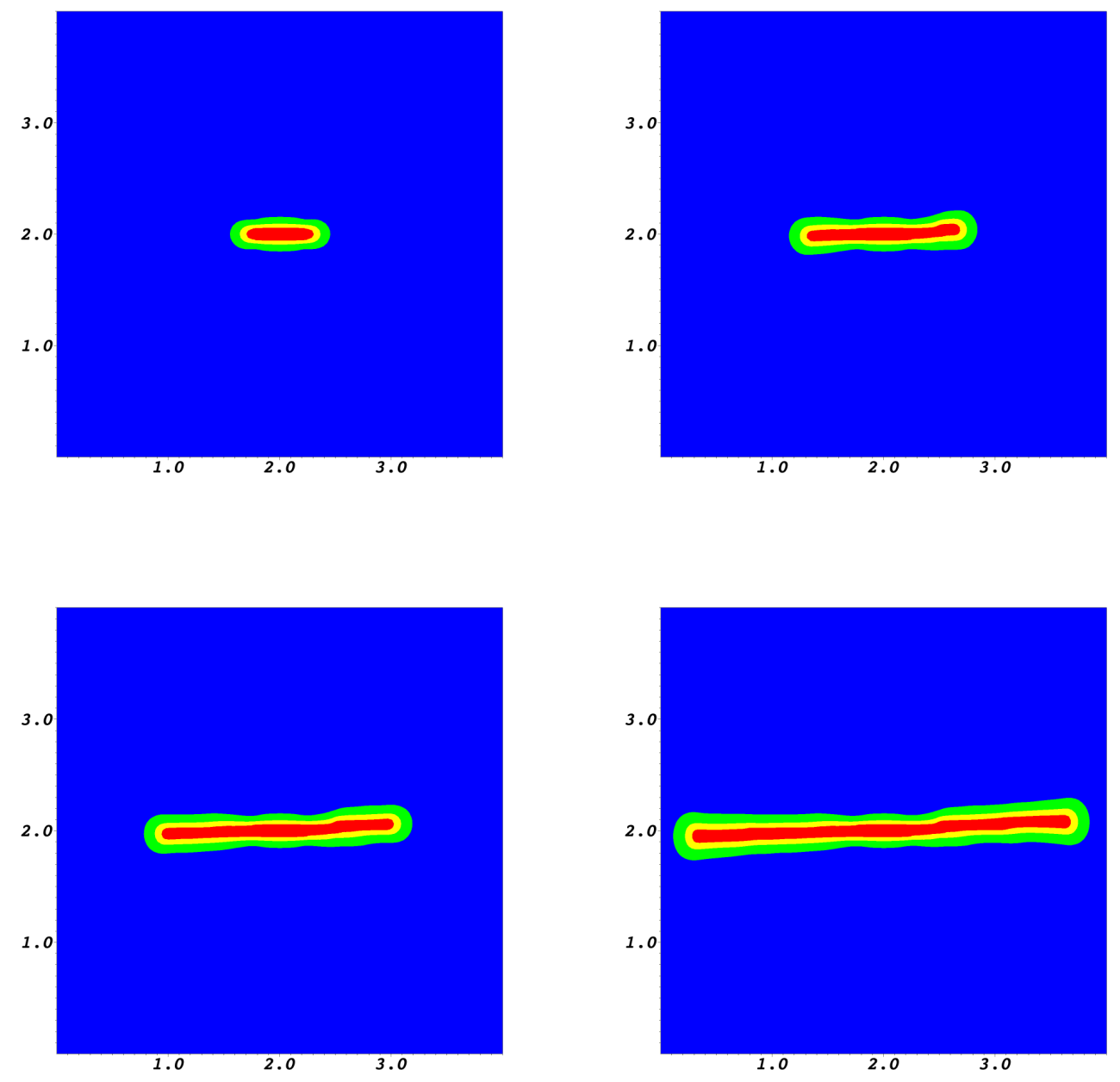

Figure 11: Example 3: Crack pattern at times $T=10,20,30,50 s$ using the phase-field variable $\varphi$. In the crack (red colored) region $\varphi=0$ and in the unbroken material, $\varphi=1$. In between $0<\varphi<1$, which denotes the smooth transition zone. 

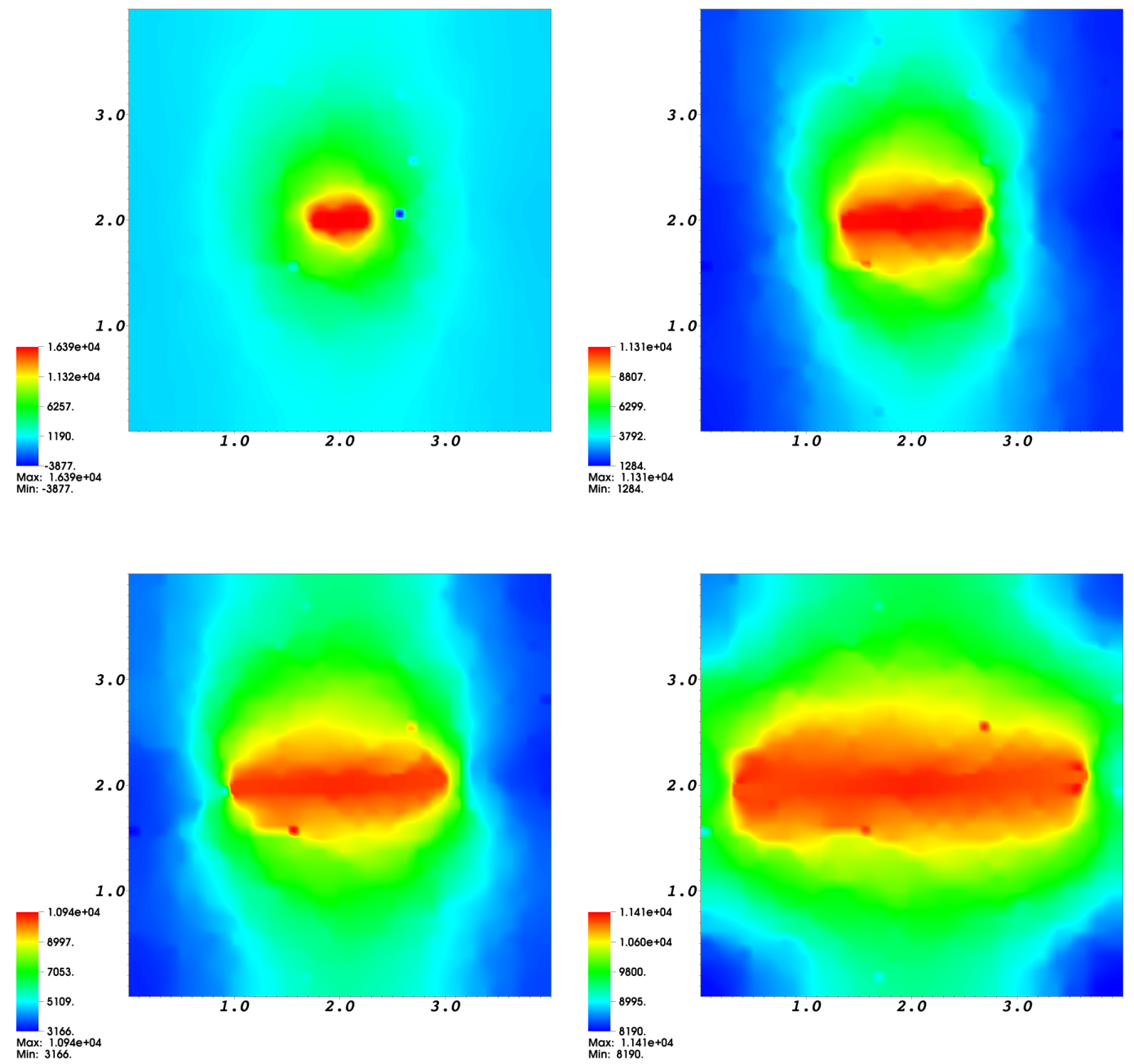

Figure 12: Example 3: Pressure (in $P a$ ) field at times $T=10,20,30,50 \mathrm{~s}$. 

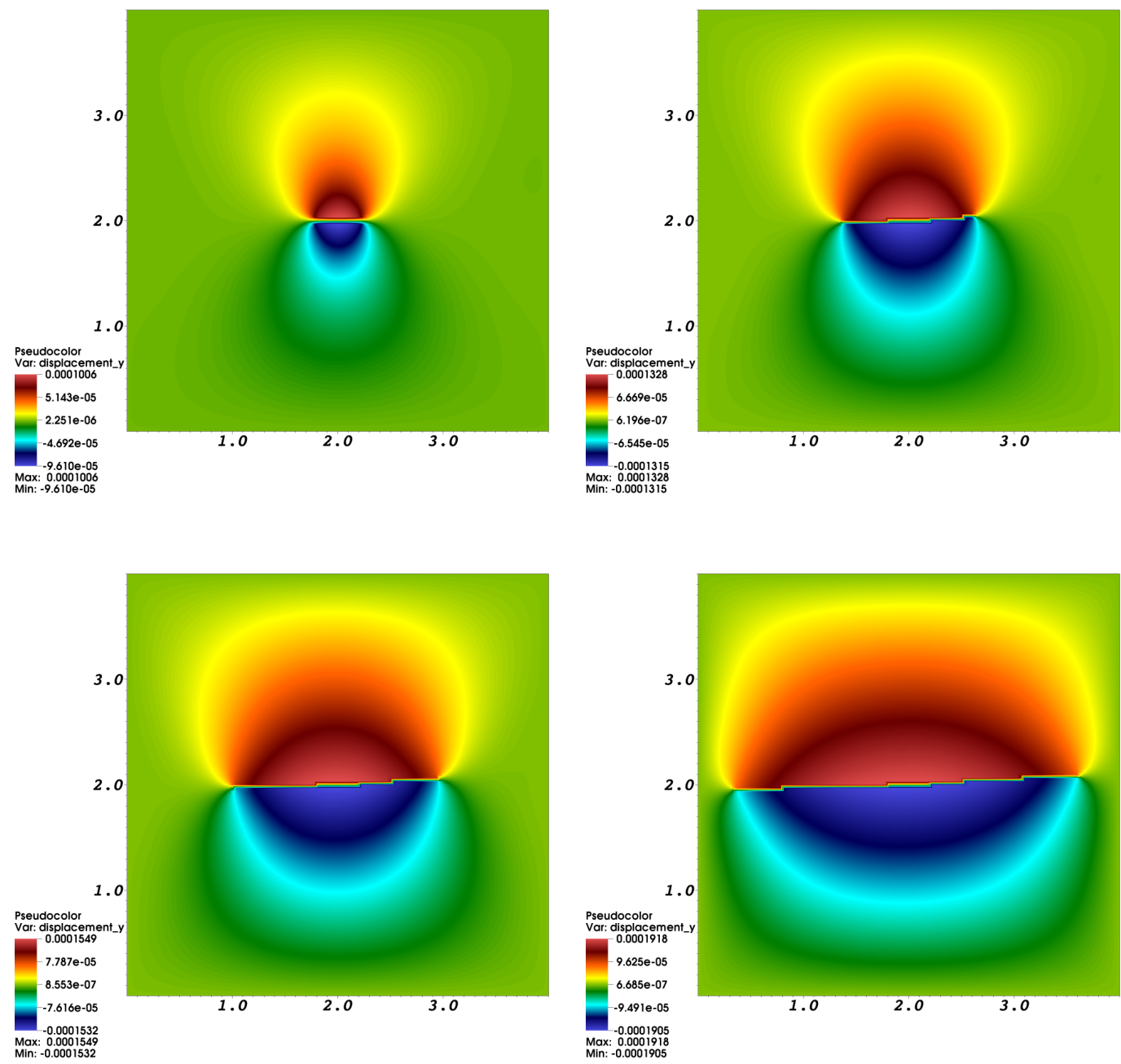

Figure 13: Example 3: $y$-displacement field (in $m$ ) at times $T=10,20,30,50 s$.

We note that the displacements are zero at the tips of the fracture and the highest value is obtained in the middle. As in the first example, this corresponds to the theoretical calculations of [20] in their Section 2.4.

\section{Conclusion}

In this paper, we formulated a fully-coupled phase-field model describing propagating cracks in a poroelastic medium. The model consists of coupled nonlinear PDEs for the displacement, the pressure and the phase field unknowns. The phase-field algorithm is based on an incremental formulation and existence of a weak solution is established. Furthermore, the corresponding Lyapunov functional, based on the free energy, is constructed. Numerical tests demonstrate the correctness of the model. Specifically, this approach can treat crack growth in heterogeneous porous media, pressurized crack evolutions and multiple interacting cracks allowing them to join and branch. Moreover, the numerical simulations confirm findings from literature that low (negative) pressures arise at fracture tips. 


\section{References}

[1] J. Adachi, E. Siebrits, A.Peirce, J. Desroches, Computer simulation of hydraulic fractures, International Journal of Rock Mechanics and Mining Sciences, 44 (2007) 739-757.

[2] W. Bangerth, T. Heister, G. Kanschat, Differential Equations Analysis Library, 2012.

[3] M.A. Biot, Mechanics of Deformation and Acoustic Propagation in Porous Media, J. Appl. Phys. 33 (1962), 1482.

[4] B. Bourdin, G.A. Francfort, J.-J. Marigo, The Variational Approach to Fracture, J. Elasticity 91 (1-3) (2008) 1-148.

[5] R. de Borst, J. Rethoré, M.A. Abellan, A numerical approach for arbitrary cracks in a fluidsaturated porous medium, Arch. Appl. Mech. 75 (2006) 595-606.

[6] R. Calhoun, M. Lowengrub, A two dimensional asymmetrical crack problem, J. Elasticity, 4 (1974) 37-50.

[7] O. Coussy, Poromechanics, John Wiley and Sons, Chichester, 2004.

[8] E.Detournay, D.I. Garagash, The near-tip region of a fluid-driven fracture propagating in a permeable elastic solid, J. Fluid Mech. 494 (2003) 1-32.

[9] G.A. Francfort, J.-J. Marigo, Revisiting brittle fracture as an energy minimization problem. J. Mech. Phys. Solids. 46 (8) (1998) 1319-1342.

[10] B. Ganis, V. Girault, M. Mear, G. Singh, M. F. Wheeler, Modeling fractures in a poro-elastic medium. Oil \& Gas Science and Technology - Rev. IFP, Energies nouvelles, Vol. 69 (2014), No. 4, pp. 515-528.

[11] F. Irzal, J. J.C. Remmers, J. M. Huyghe, R. de Borst, A large deformation formulation for fluid flow in a progressively fracturing porous material, Comput. Methods Appl. Mech. Engrg. 256 (2013) 29-37.

[12] C. Miehe, F. Welschinger, M. Hofacker, Thermodynamically consistent phase-field models of fracture: variational principles and multi-field FE implementations, International Journal of $\mathrm{Nu}$ merical Methods in Engineering, 83 (2010) 1273-1311.

[13] T. Heister, M. F. Wheeler, T. Wick, A primal-dual active set method and predictor-corrector mesh adaptivity for computing fracture propagation using a phase-field approach, Comput. Methods Appl. Mech. Engrg., Vol. 290, pp. 466-495 (2015).

[14] M. Hintermüller, K. Ito, K. Kunisch, The primal-dual active set strategy as a semismooth Newton method, SIAM Journal on Optimization, Vol. 13 (3), pp. 865-888 (2002).

[15] A. Mikelić, M. F. Wheeler, T. Wick, A phase-field approach to the fluid filled fracture surrounded by a poroelastic medium, 2013, ICES Report 13-15.

[16] A. Mikelić, M. F. Wheeler, T. Wick, A quasistatic phase field approach to pressurized fractures, Nonlinearity, 28 (2015), 1371-1399.

[17] A. Mikelić, M. F. Wheeler, T. Wick, A phase-field method for propagating fluid-filled fractures coupled to a surrounding porous medium, SIAM Multiscale Model. Simul., 13 (2015) 367-398. 
[18] S. Secchi and B. A. Schrefler, A method for 3-D hydraulic fracturing simulation, Int J Fract , 178 (2012) 245-258.

[19] B. A. Schrefler and St. Secchi and L. Simoni, On adaptive refinement techniques in multi-field problems including cohesive fracture, Comput. Meth. Appl. Mech. Engrg., 195 (2006) 444-461.

[20] I. N. Sneddon, M. Lowengrub, Crack problems in the classical theory of elasticity. The SIAM series in Applied Mathematics. John Wiley and Sons (1969).

[21] I. Tolstoy, ed., Acoustics, elasticity, and thermodynamics of porous media. Twenty-one papers by M.A. Biot, Acoustical Society of America, New York, 1992.

[22] M.F. Wheeler, T. Wick, W. Wollner, An augmented-Lagrangian method for the phase-field approach for pressurized fractures, Comput. Methods Appl. Mech. Engrg. 271 (2014) 69-85.

[23] M. A. Heroux, R. A. Bartlett, V. E. Howle, R. J. Hoekstra, J. J. Hu, T. G. Kolda, R. B. Lehoucq, K. R. Long, R. P. Pawlowski, E. T. Phipps, A. G. Salinger, H. K. Thornquist, R. S. Tuminaro, J. M. Willenbring, A. Williams, K. S. Stanley, An overview of the trilinos project. ACM Trans. Math. Softw. 31 (2005) 397-423.

[24] T. Wick, G. Singh, M.F. Wheeler, Pressurized-fracture propagation using a phase-field approach coupled to a reservoir simulator, SPE HFTC meeting in the Woodlands, SPE 168597-MS. 